Surface Water Data at

Los Alamos National Laboratory:

1995 Water Year

D. A. Shaull

M. R. Alexander

R. P. Reynolds

ong 


\section{DISCLAIMER}

Portions of this document may be illegible in electronic image products. Images are produced from the best available original document. 


\section{DISCLAIMER}

This report was prepared as an account of work sponsored by an agency of the United States Government. Neither the United States Government nor any agency thereof, nor any of their employees, make any warranty, express or implied, or assumes any legal liability or responsibility for the accuracy, completeness, or usefulness of any information, apparatus, product, or process disclosed, or represents that its use would not infringe privately owned rights. Reference herein to any specific commercial product, process, or service by trade name, trademark, manufacturer, or otherwise does not necessarily constitute or imply its endorsement, recommendation, or favoring by the United States Government or any agency thereof. The views and opinions of authors expressed herein do not necessarily state or reflect those of the United States Government or any agency thereof. 


\section{CONTENTS}

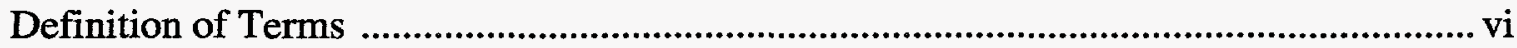

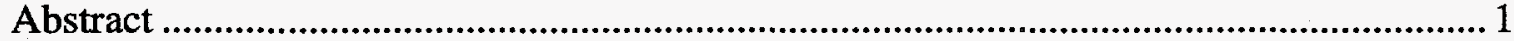

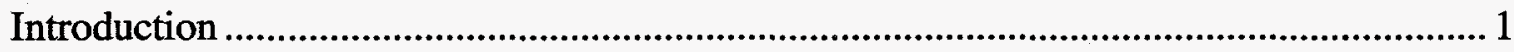

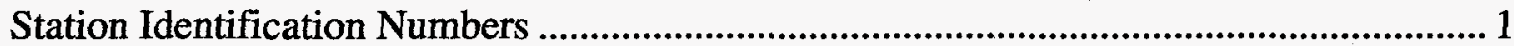

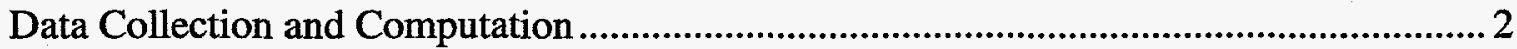

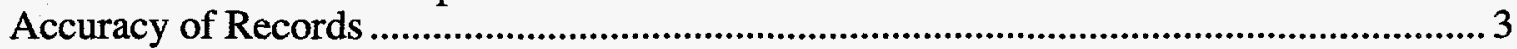

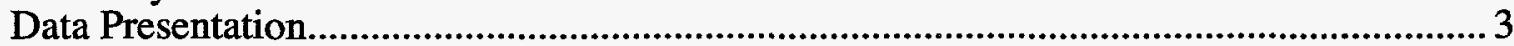

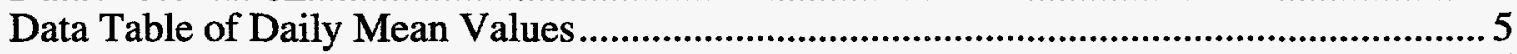

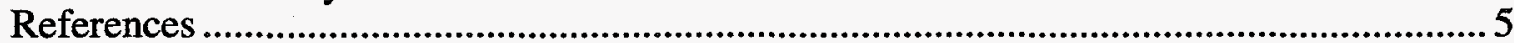

TABLES AND ILLUSTRATIONS

Gaging Stations at Los Alamos National Laboratory .............................................. 6

Summary of Discharges from Stream Monitoring Stations at Los Alamos National Laboratory ..................................................................... 7

Los Alamos Canyon Seepage/Channel Loss Investigation ........................................ 41

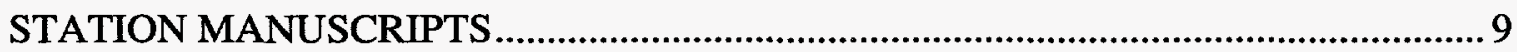

08313025 Los Alamos Canyon at Los Alamos, NM ................................................ 11

08313030 Los Alamos Canyon below TA-2 near Los Alamos, NM.............................. 13

08313042 Los Alamos Canyon near Los Alamos, NM ............................................ 15

08313060 Pueblo Canyon near Los Alamos, NM...................................................... 17

08313125 Sandia Canyon above Highway 4 near White Rock, NM............................ 19

08313200 Mortandad Canyon at Laboratory Technical Area (TA)-50

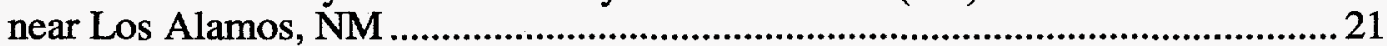

08313204 Mortandad Canyon at Laboratory Boundary.............................................. 23

08313225 Cañada del Buey above White Rock, NM ..................................................... 25

08313230 Cañada del Buey at White Rock, NM ........................................................2 27

08313240 Parajito Canyon above Highway 501 near Los Alamos, NM .......................... 29

08313245 Parajito Canyon above TA-18 near Los Alamos, NM ...................................... 31

08313250 Parajito Canyon above Highway 4 near White Rock, NM ............................ 33

08313252 Water Canyon above Highway 501 near Los Alamos, NM......................... 35

08313253 Cañon del Valle above Highway 501 near Los Alamos, NM.......................... 37

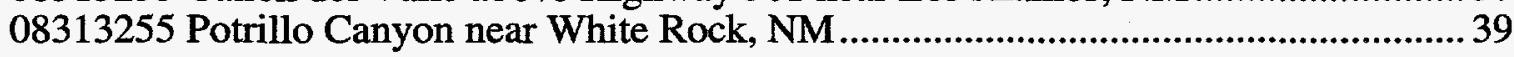




\section{DEFINITION OF TERMS}

Acre-foot (AC-FT, acre-ft) is the quantity of water required to cover one acre to a depth of one foot and is equivalent to 43,560 cubic feet or 325,851 gallons or $1,233.49$ cubic meters.

Cfs-day is the volume of water represented by the flow of one cubic foot per second for 24 hours. It is equivalent to 86,400 cubic feet, 1.98347 acre-feet, 646,317 gallons, or 2,445 cubic meters.

Control designates a feature downstream from the gage that determines the stage-discharge relation at the gage. This feature may be a natural constriction of the channel, an artificial structure, or a uniform cross section over a long reach of the channel.

Control structure as used in this report is a structure on a stream or canal that is used to regulate the flow or stage of the stream or to prevent the intrusion of salt water.

Cubic feet per second per square mile $\left[\left(\mathrm{ft}^{3} / \mathrm{s}\right) / \mathrm{mi}^{2}\right]$ is the average number of cubic feet of water flowing per second from each square mile of area drained, assuming that the runoff is distributed uniformly in time and area.

Cubic foot per second $\left(\mathrm{ft}^{3} / \mathrm{s}, \mathrm{ft}^{3} / \mathrm{s}, \mathrm{cfs}\right)$ is the rate of discharge representing a volume of one cubic foot passing a given point during one second and is equivalent to 7.48 gallons per second or 448.8 gallons per minute or 0.02832 cubic meters per second.

Discharge is the volume of water (or more broadly, volume of fluid plus suspended sediment) that passes a given point within a given period of time.

Mean discharge (MEAN) is the arithmetic mean of individual daily mean discharges during a specific period.

Instantaneous discharge is the discharge at a particular instant of time.

Drainage area of a stream at a specified location is that area, measured in a horizontal plane, enclosed by a topographic divide, from which direct surface runoff from precipitation normally drains by gravity into the stream above the specified point. Figures of drainage area given herein include all closed basins, or noncontributing areas, within the area unless otherwise noted.

Drainage basin is a part of the surface of the earth that is occupied by a drainage system, which consists of a surface stream or a body of impounded surface water together with all tributary surface streams and bodies of impounded surface water.

Gage height (G.H.) is the water-surface elevation referred to some arbitrary gage datum. Gage height is often used interchangeably with the more general term "stage," although gage height is more appropriate when used with a reading on a gage.

Gaging station is a particular site on a stream, canal, lake, or reservoir where systematic observations of hydrologic data are obtained.

National Geodetic Vertical Datum of 1929 (NGVD) is a geodetic datum derived from a general adjustment of the first order level nets of both the United States and Canada. It was formerly called Sea Level Datum of 1929 or "mean sea level" in this series of reports. Although the datum was derived from the average sea level over a period of many years at 26 tide stations along the 
Atlantic, Gulf of Mexico, and Pacific Coasts, it does not necessarily represent local mean sea level at any particular place.

Water year in Geological Survey reports dealing with surface water supply is the 12-month period, October 1 through September 30. The water year is designated by the calendar year in which it ends and which includes 9 of the 12 months. Thus, the year ending September 30, 1980, is called the "1980 water year."

WDR is used as an abbreviation for "Water-Data Report" in the REVISED RECORDS paragraph to refer to State annual hydrologic-data reports (WRD was used as an abbreviation for "Water-Resources Data" in reports published prior to 1976).

WSP is used as an abbreviation for "Water-Supply Paper" in references to previously published reports. 


\title{
SURFACE WATER DATA AT LOS ALAMOS NATIONAL LABORATORY: 1995 WATER YEAR
}

\author{
D. A. Shaull, M. R. Alexander, and R. P. Reynolds
}

\begin{abstract}
The principle investigators collected and computed surface water discharge data from 15 streamgaging stations that cover most of Los Alamos National Laboratory. The United States Department of Interior Geological Survey, Water Resources Division, operates two of the stations under a subcontract; these are identified in the station manuscripts. Included in this report are data from one seepage run conducted in Los Alamos Canyon during the 1995 water year.
\end{abstract}

\section{INTRODUCTION}

This first annual water data report from Los Alamos National Laboratory (LANL, Laboratory) contains flow data from 15 stream-gaging stations that cover most of the Laboratory's property. We placed particular emphasis on the Laboratory's downstream boundary, which is approximated by the route taken by New Mexico State Highway 4 . The upstream boundary is approximated by New Mexico State Highway 501. Some of the gaging stations are within Laboratory boundaries.

The Water Quality and Hydrology Group (ESH-18) developed and installed the stream-gaging network; the US Department of Interior, Geological Survey, Water Resources Division (USGSWRD) did some of the contract work. USGS-WRD designed and installed the necessary data collection structures. Construction took place in 1993 and 1994, and USGS-WRD operated the network until January 1, 1995, when ESH-18 assumed operational responsibility. Groups other than ESH-18 also conduct site-specific earth science research using data from these stations.

\section{STATION IDENTIFICATION NUMBERS}

USGS-WRD assigns a unique identification number to each stream-gaging station it establishes. All sites numbered since 1950 are part of the downstream order system. The downstream order system increases station numbers in the downstream direction along main streams, and in the case of this report, their respective mouths to the Rio Grande. 


\section{DATA COLLECTION AND COMPUTATION}

A complete record-gaging station gathers records of stage and discharge measurements from streams or canals. In addition to these stage and discharge measurements, we directly observe factors affecting the stage/discharge relation, consult weather records, and use other information that supplements base data in determining daily flow. Direct readings on a nonrecording gage or from the data logger provide continuous records of stage. We measure discharge with current meters, using methods adapted by the Geological Survey as a result of experience accumulated since 1880. Standard textbooks describe these methods as doWater-Supply Paper 2175 and the US Geological Survey Technique of Water Resources Investigations, Book 3, Chapter A6.

We use stage/discharge relation curves to prepare rating tables that give the discharge for any stage measured at a stream-gaging station. When necessary to define discharge extremes outside the range of current meter measurements, we extend the curves using

- logarithmic plotting,

- velocity area studies,

- results of indirect measurements of peak discharge, such as slope area or contracted opening measurements and computations of flow over dams or weirs, or

- step backwater techniques.

Daily mean stages (gage heights) are applied to the stage-discharge curves or tables to compute daily mean discharges. If the stage/discharge relation is subject to change because of frequent or continual change in the physical features that form the control, the daily mean discharge is computed by the shifting-control method. In the shifting-control method, correction factors based on individual discharge measurements and notes by personnel taking the measurements are applied to the gage heights before discharges are determined from the curves or tables.

The shifting-control method is also used if the stage/discharge relation for a station is temporarily changed by the presence of aquatic growth or debris on the control. At some northern streamgaging stations, the stage/discharge relation is affected by ice in the winter and it becomes impossible to compute discharge in the usual manner. Discharge for period of ice effect is computed on the basis of gage height record and occasional winter discharge measurements. Consideration is given to the available information on temperature and precipitation, notes of observations, and comparable discharge records for other stations in the same or nearby basins for comparable periods of time. 
For some gaging stations, periods occur when no gage height record is obtained, or the recorded gage height is so faulty that it cannot be used to compute daily discharge or contents. This happens when the recorder stops or otherwise fails to operate properly, intakes are plugged, the float is frozen in the well, etc. For such periods, the daily discharges are estimated on the basis of recorded range-in-stage, prior and subsequent records, discharge measurements, weather records, and record comparison made against other stations in the same or nearby basins. Likewise, daily contents may be estimated from operator logs, prior and subsequent records, inflow-outflow studies, and other information.

\section{ACCURACY OF RECORDS}

Two factors determine the accuracy of streamflow records:

- stability of the stage-discharge relation, or if the control is unstable, the frequency of discharge measurements; and

- accuracy of measurements or stage, accuracy of discharge measurements, and interpretations of records.

Accuracy attributed to records is noted under "Remarks."

- Excellent-95\% of daily discharges are within 5\% of true value;

- Good-95\% of daily discharges are within $10 \%$ of true value;

- Fair- $95 \%$ of daily discharges are within $15 \%$ of true value; and

- Poor-records do not meet the criteria mentioned.

Differences in accuracy may be attributed to different parts of a given record.

The number of significant figures used to report daily mean discharges is based solely on the magnitude of the discharge value:

\begin{tabular}{|l|l|}
\hline \multicolumn{1}{|c|}{ If - the value $(\mathrm{cfs})$ is } & Then-its reported to \\
\hline less than $1 \mathrm{cfs}$ & nearest hundredth \\
\hline $1-10 \mathrm{cfs}$ & nearest tenth \\
\hline $10-1000 \mathrm{cfs}$ & whole number \\
\hline above $1000 \mathrm{cfs}$ & three significant figures \\
\hline
\end{tabular}




\section{DATA PRESENTATION}

The records published in this report are for each gaging station and comprise two parts:

- Station manuscript description with photo and

- Data table for the water year (October 1, 1994 to September 30, 1995).

The station manuscript provides data under various headings: station location, period of record, average discharge, historical extremes, record accuracy, and other points pertinent to station operation and regulation. Each continuous record of discharge includes the following categories of descriptions.

Location. The most accurate and available maps provide location information. The location of the gage with respect to the vicinity's cultural and physical features is given as well as a name that makes reference to place. For a few stations, the US Army Corps of Engineers or the Water Resources Council (River Mileage Measurement, Bulletin 14, rev. October 1968) provided river milleage. We define left and right banks from the perspective of facing downstream.

Drainage area. The most accurate and available maps provide drainage area measurements. The accuracy of drainage area measurements varies depending on the type of map available for this purpose.

Period of record. The period of record is the time during which published records exist for a station or its equivalent station. An equivalent station is one that was in operation at a time that the present station was not and was located so that records from it can reasonably be considered equivalent with records from the present station.

Gage. This section describes the type of gage in current use. The datum of the current gage referred to in the National Geodetic Vertical Datum of 1929 (see glossary) and a condensed history of the types, locations, and datums of previous gages are given under this heading.

Remarks. The date in the station description for water discharge records identifies all periods of estimated daily discharge records. The text also presents information relative to the accuracy of the records, special methods of computation, conditions that affect natural flow at the station, and other pertinent information. 
Extremes for period of record. Extremes may include maximum and minimum stages and maximum and minimum discharges or content. Unless otherwise qualified, the maximum discharge or content is the instantaneous maximum corresponding to the highest stage that occurred. The highest stage may have been obtained from a graphic or digital recorder, a crest stage gage, or by direct observation of a nonrecording gage. If the maximum stage did not occur on the same day as the maximum discharge or content, it is given separately. Similarly, the minimum is the instantaneous minimum discharge, unless otherwise qualified, and was determined and is reported in the same manner as the maximum.

Extremes outside period of record. This section contains information concerning major floods or unusually low flows that occurred outside the stated period of record. The information may have been obtained from other agencies, old data files, newspapers, or local citizens.

Extremes for current year. Extremes given here are similar to those for the period of record. The time for occurrence of peaks is expressed in 24-hour local standard time. For example, 12:30 A.M. is 0030 and 1:30 P.M. is 1330 . The minimum for the current water year appears in this section.

\section{DATA TABLE OF DAILY MEAN VALUES}

The daily table of discharge records for stream gaging stations gives the mean discharge for each day of the water year. In the monthly summary for the table, the line headed "Total" gives the sum of the daily figures for each month; the line headed "Mean" gives the average flow in cubic feet per seond for the month; and the lines headed "Max" and "Min" give the maximum and minimum daily mean discharges for each month and in acre feet, respectively, in the line headed "AC-FT."

\section{REFERENCES}

Water-Supply Paper 2175 and the US Geological Survey Technique of Water Resources Investigations, Book 3, Chapter A6.

US Army Corps of Engineers, River Mileage Measurement, Bulletin 14, rev. October 1968. National Geodetic Vertical Datum of 1929. 


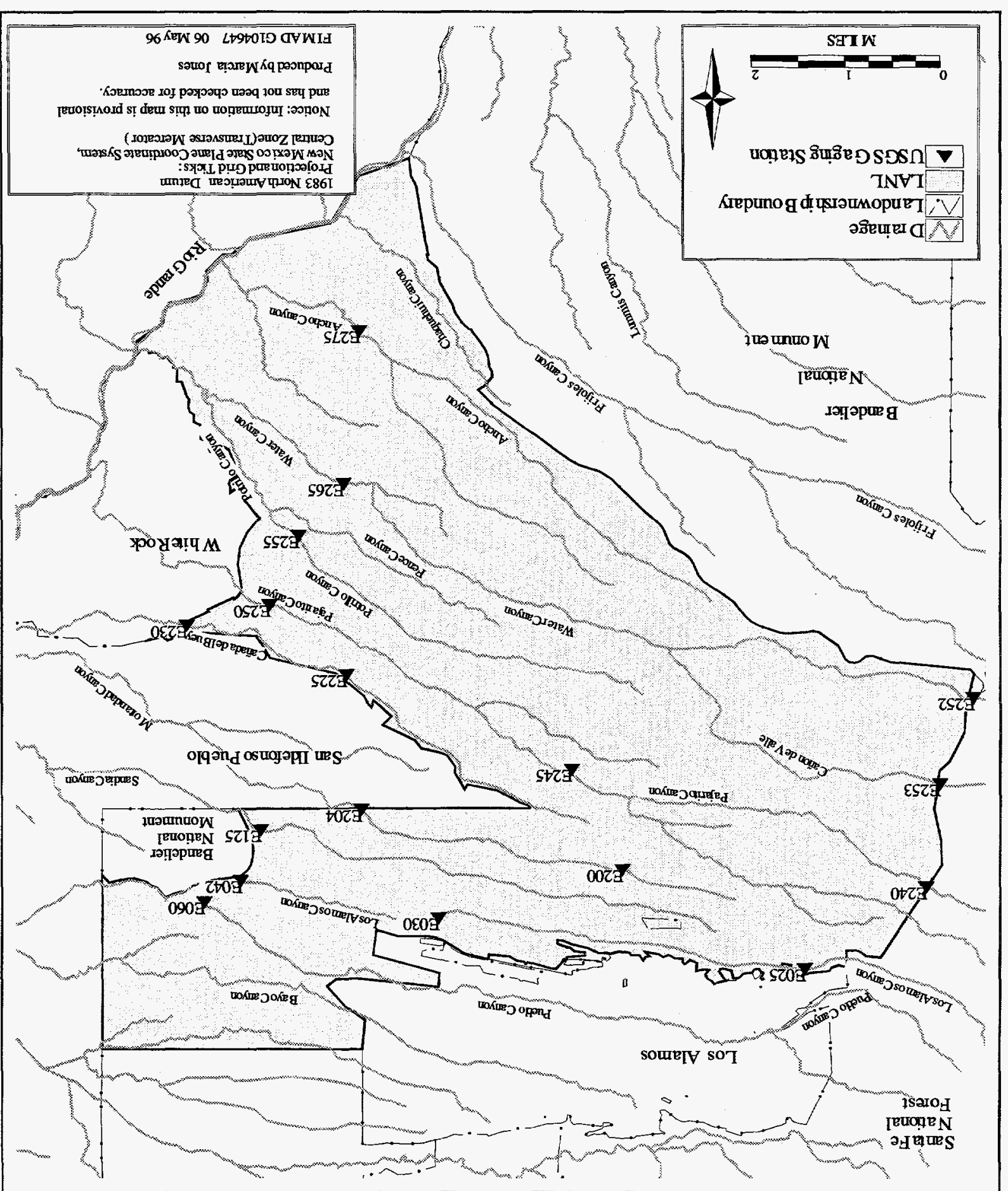

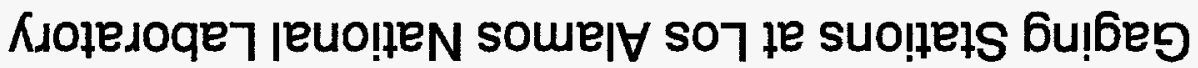




\section{Summary of Discharges from Stream Monitoring Stations at Los Alamos National Laboratory}

Water Year 1995

October 1, 1994-September 30, 1995

\begin{tabular}{|c|c|c|c|c|c|c|}
\hline \multirow[t]{2}{*}{ Canyon Sites } & \multirow{2}{*}{$\begin{array}{l}\text { Days w/ } \\
\text { Flow }\end{array}$} & \multicolumn{2}{|c|}{ Total volume of Water } & \multicolumn{2}{|c|}{ Instantaneous Max } & \multirow[t]{2}{*}{ Comments } \\
\hline & & AC-FT & Gallons & $\mathrm{FT}^{3} / \mathrm{s}$ & GPM & \\
\hline $\begin{array}{l}\text { E025 Upper Los } \\
\text { Alamos }\end{array}$ & 247 & 465.0 & $151,520,715$ & 10.0 & 4,488 & \\
\hline $\begin{array}{l}\text { E030 Middle Los } \\
\text { Alamos }\end{array}$ & 169 & 492.0 & $160,318,692$ & 12.0 & 5,386 & \\
\hline $\begin{array}{l}\text { E042 Lower Los } \\
\text { Alamos** }\end{array}$ & 110 & 328.0 & $106,879,128$ & 54.0 & 24,235 & USGS operated \\
\hline E060 Pueblo** & $\underline{365}$ & 874.0 & $28,481,038$ & 6.3 & 2,621 & USGS operated \\
\hline E125 Sandia** & 6 & 5.0 & $1,629,255$ & 13.0 & 5,834 & \\
\hline $\begin{array}{l}\text { E204 Lower } \\
\text { Mortandad** }\end{array}$ & 0 & 0.0 & - & 0.0 & - & \\
\hline $\begin{array}{l}\text { E200 Middle } \\
\text { Mortandad }\end{array}$ & 83 & 18.0 & $5,865,318$ & 9.7 & 4,353 & Record began 5/10/95 \\
\hline $\begin{array}{l}\text { E225 Upper Cañada } \\
\text { del Buey }\end{array}$ & 1 & 0.4 & 130,340 & 17.0 & 7,630 & \\
\hline $\begin{array}{l}\text { E230 Lower Cañada } \\
\text { del Buey** }\end{array}$ & 15 & 14.0 & $4,561,914$ & 75.0 & 33,660 & \\
\hline E240 Upper Pajarito & 239 & 106.0 & $34,540,206$ & 1.9 & 853 & \\
\hline E245 Middle Pajarito & 211 & 250.0 & $81,462,750$ & 24.0 & 10,771 & \\
\hline $\begin{array}{l}\text { E250 Lower } \\
\text { Pajarito** }\end{array}$ & 210 & 30.0 & $9,775,530$ & 4.6 & 2,064 & \\
\hline E255 Potrillo & 3 & 3.5 & $1,140,479$ & 63.0 & 28,274 & \\
\hline E252 Upper Water & 74 & 9.5 & $3,095,585$ & 0.21 & 94 & \\
\hline E253 Cañon de Valle & 0 & & & & & \\
\hline $\begin{array}{l}\text { E265 Lower Water ** } \\
\text { *** }\end{array}$ & 2 & & & 0.21 & 9,425 & $\begin{array}{l}\text { Gage rating to be } \\
\text { established }\end{array}$ \\
\hline E275 Ancho & 5 & & & & & $\begin{array}{l}\text { Gage rating to be } \\
\text { established }\end{array}$ \\
\hline
\end{tabular}

USGS1,3/18/96

** Station at downstream Laboratory boundary

*** Daily values table not published this year 
STATION MANUSCRIPTS 


\section{Los Alamos CaNyon at Los Alamos, NM}

LOCATION. Lat $35^{\circ} 52^{\prime} 48^{\prime \prime}$, long $106^{\circ} 19^{\prime} 42^{\prime \prime}$, in SE 1/4 SE $1 / 4$ sec. 17, T. 19 N., R. 6 E., Los Alamos County, on right bank 1.5 mi downstream from Los Alamos Reservoir, and $0.4 \mathrm{mi}$ upstream from "Rainbow" bridge on Diamond Drive over Los Alamos Canyon.

DRAINAGE AREA. $7.12 \mathrm{mi}^{2}$.

PERIOD OF RECORD. October 1, 1993 through September 30, 1995.

GAGE. Data logger with cellular telemetry and 2-ft Parshall Flume. Elevation of gage is 7,220 ft above National Geodetic Vertical Datum of 1929 from topographic map.

REMARKS. Water discharge records good. Flow partially controlled by Los Alamos Reservoir 1.5 mi upstream.

EXTREMES FOR PERIOD OF RECORD. Maximum discharge $10 \mathrm{cfs}$, May 4, 1995; gage height $1.26 \mathrm{ft}$. No flow at times.

EXTREMES FOR CURRENT WATER YEAR. Maximum discharge $10 \mathrm{cfs}$, at $1035 \mathrm{hrs,} \mathrm{May} \mathrm{4,}$ 1995; gage height $1.26 \mathrm{ft}$. No flow at times.

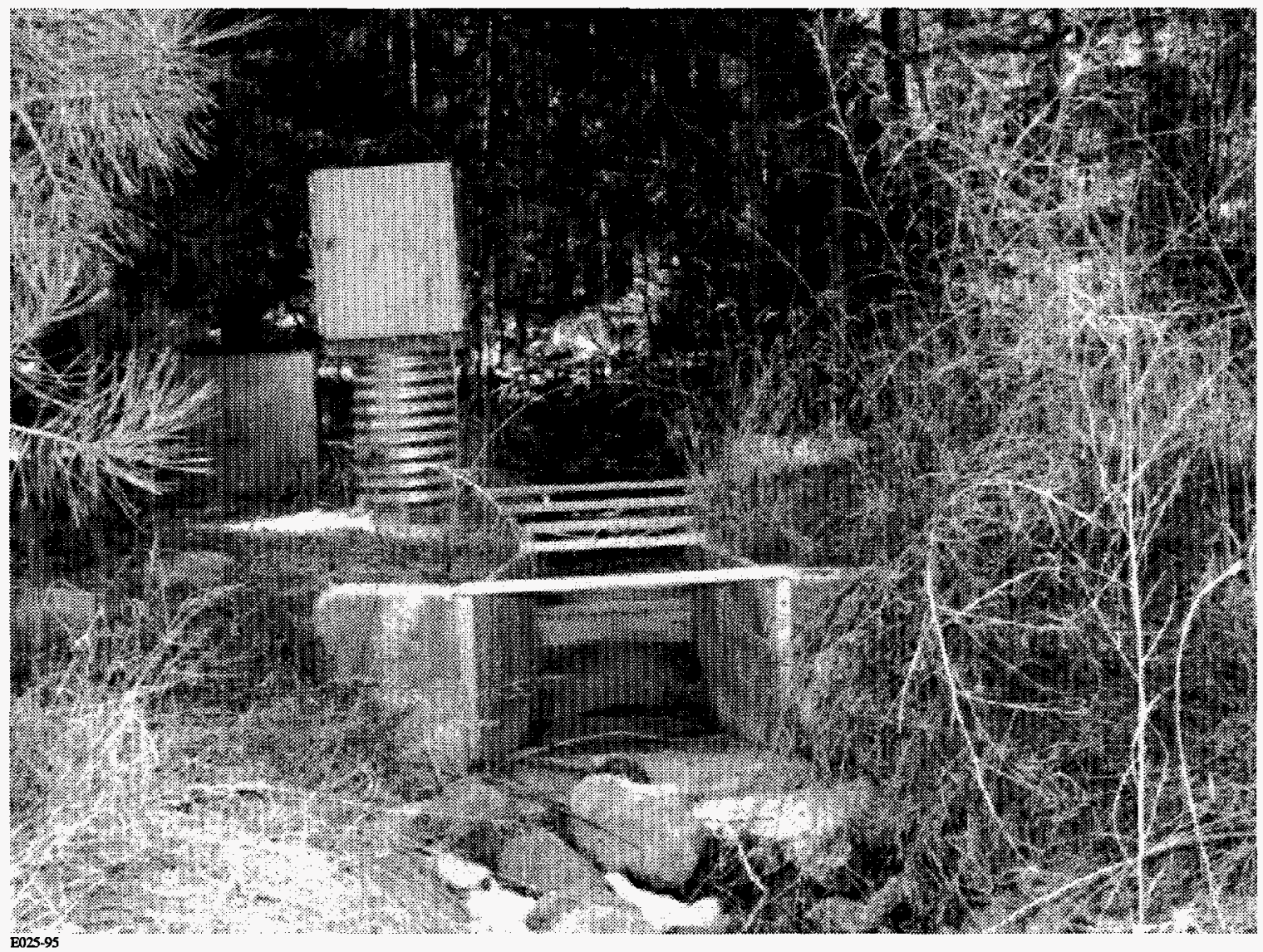




\section{Los Alamos Canyon at los Alamos, NM}

\section{DAILY DISCHARGE IN CUBIC FEET PER SECOND \\ WATER YEAR OCTOBER 1994 TO SEPTEMBER 1995}

\begin{tabular}{|c|c|c|c|c|c|c|c|c|c|c|c|c|}
\hline Day & OCT & NOV & DEC & JAN & FEB & MAR & APR & MAY & JUN & JUL & AUG & SEP \\
\hline 1 & 0 & 0.20 & 1.1 & 0 & 0 & 0.38 & 0.63 & 5.4 & 1.4 & 0 & 0.13 & 0.37 \\
\hline 2 & 0 & 0.20 & 1.1 & 0 & 0 & 0.32 & 0.58 & 5.3 & 1.3 & 0 & 0.13 & 0.31 \\
\hline 3 & 0 & 0.26 & 1.1 & 0 & 0 & 0.41 & 0.54 & 6.2 & 1.2 & 0 & 0.13 & 0.24 \\
\hline 4 & 0 & 0.24 & 1.1 & 0 & 0 & 0.49 & 0.51 & 5.8 & 1.1 & 0 & 0.13 & 0.18 \\
\hline 5 & 0 & 0.22 & 0.91 & 0 & 0 & 0.55 & 0.47 & 4.3 & 0.97 & 0 & 0.11 & 0.12 \\
\hline 6 & 0 & 0.23 & 0.07 & 0 & 0 & 1.2 & 0.48 & 4.2 & 0.84 & 0 & 0.10 & 0.17 \\
\hline 7 & 0 & 0.22 & 0.01 & 0 & 0 & 1.6 & 0.55 & 3.8 & 0.75 & 0 & 0.10 & 0.13 \\
\hline 8 & 0 & 0.23 & 0.03 & 0 & 0 & 1.6 & 0.66 & 3.4 & 0.66 & 0 & 0.10 & 0.15 \\
\hline 9 & 0 & 0.20 & 0.08 & 0 & 0 & 1.3 & 0.91 & 2.8 & 0.59 & 0 & 0.10 & 0.28 \\
\hline 10 & 0 & 0.20 & 0 & 0 & 0 & 1.1 & 1.1 & 2.6 & 0.57 & 0 & 0.10 & 0.27 \\
\hline 11 & 0 & 0.24 & 0 & 0 & 0 & 0.95 & 0.97 & 2.7 & 0.50 & 0 & 0.16 & 0.38 \\
\hline 12 & 0 & 2.5 & 0 & 0 & 0 & 0.91 & 0.86 & 3.2 & 0.42 & 0 & 0.20 & 0.29 \\
\hline 13 & 0 & 4.1 & 0 & 0 & 0 & 0.88 & 0.77 & 3.2 & 0.34 & 0 & 0.18 & 0.20 \\
\hline 14 & 0.01 & 2.9 & 0 & 0 & 0 & 0.88 & 0.82 & 3.0 & 0.32 & 0 & 0.25 & 0.23 \\
\hline 15 & 0.05 & 2.0 & 0 & 0 & 0 & 0.88 & 0.98 & 3.0 & 0.29 & 0 & 0.19 & 0.19 \\
\hline 16 & 0.23 & 1.4 & 0 & 0 & 0 & 0.99 & 1.0 & 3.0 & 0.29 & 0 & 0 & 0.17 \\
\hline 17 & 0.40 & 0.96 & 0 & 0 & 0 & 1.2 & 1.2 & 3.9 & 0.9 & 0 & 0 & 0.15 \\
\hline 18 & 0.37 & 0.73 & 0 & 0 & 0 & 1.3 & 1.1 & 4.1 & 0.9 & 0 & 0 & 0.09 \\
\hline 19 & 0.33 & 0.63 & 0 & 0 & 0 & 1.5 & 1.1 & 3.4 & 0.64 & 0 & 0 & 0.02 \\
\hline 20 & 0.30 & 0.57 & 0 & 0 & 0 & 1.7 & 0.98 & 2.9 & 0.58 & 0.26 & 0.11 & 0.01 \\
\hline 21 & 0.28 & 0.53 & 0 & 0 & 0 & 2.0 & 0.94 & 2.5 & 0.52 & 0.26 & 0.02 & 0 \\
\hline 22 & 0.27 & 0.48 & 0 & 0 & 0.01 & 2.4 & 1.0 & 2.2 & 0.45 & 0.23 & 0 & 0.01 \\
\hline 23 & 0.25 & 0.44 & 0 & 0 & 0.04 & 2.2 & 0.81 & 1.6 & 0.40 & 0.21 & 0.01 & 0 \\
\hline 24 & 0.26 & 0.4 & 0 & 0 & 0.06 & 1.9 & 0.77 & 2.1 & 0.43 & 0.20 & 0.40 & 0 \\
\hline 25 & 0.26 & 0.36 & 0 & 0 & 0.14 & 1.8 & 0.86 & 1.8 & 0.46 & 0.20 & 0.63 & 0 \\
\hline 26 & 0.26 & 0.33 & 0 & 0 & 0.21 & 1.5 & 1.3 & 1.7 & 0.43 & 0.18 & 0.82 & 0 \\
\hline 27 & 0.26 & 0.31 & 0 & 0 & 0.25 & 1.3 & 1.7 & 1.5 & 0.37 & 0.17 & 0.91 & 0 \\
\hline 28 & 0.23 & 1.1 & 0 & 0 & 0.32 & 1.1 & 2.4 & 1.4 & 0.33 & 0.17 & 0.68 & 0 \\
\hline 29 & 0.23 & 1.1 & 0 & 0 & L & 0.95 & 3.4 & 1.8 & 0.31 & 0.17 & 0.72 & 0.02 \\
\hline 30 & 0.22 & 1.1 & 0 & 0 & - & 0.80 & 4.8 & 1.6 & 0.39 & 0.17 & 0.58 & 0.01 \\
\hline 31 & 0.20 & $\ldots$ & 0 & 0 & - & 0.70 & 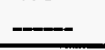 & 1.5 & - & 0.14 & 0.46 & - \\
\hline Total & 4.41 & 24.38 & 5.5 & 0 & 1.03 & 36.79 & 34.19 & 95.9 & 18.65 & 2.36 & 7.45 & 3.99 \\
\hline Mean & 0.14 & 0.81 & 0.18 & 0 & 0.037 & 1.19 & 1.14 & 3.09 & 0.62 & 0.076 & 0.24 & 0.13 \\
\hline Max & 0.4 & 4.1 & 1.1 & 0 & 0.32 & 2.4 & 4.8 & 6.2 & 1.4 & 0.26 & 0.91 & 0.38 \\
\hline Min & 0 & 0.2 & 0 & 0 & 0 & 0.32 & 0.47 & 1.4 & 0.29 & 0 & 0 & 0 \\
\hline AC-FT & 8.7 & 48 & 11 & 0 & 2 & 73 & 68 & 190 & 37 & 4.7 & 15 & 7.9 \\
\hline
\end{tabular}

CY 1994: TOTAL *34.29 MEAN 0.37 MAX 4.1 MIN 0 AC-FT 68

WATER YEAR 1995: TOTAL 234.65 MEAN 0.64 MAX 6.2 MIN 0 AC-FT 465

* Incomplete Record 


\section{Los Alamos Canyon below TA-2 NeAR LOS Alamos, NM}

LOCATION. Lat $35^{\circ} 52^{\prime} 20^{\prime \prime}$, long $106^{\circ} 15^{\prime} 37^{\prime \prime}$, SW1/4, SE1/4 sec. 14 , T. 19 N, R.6 E., Los Alamos County, $150 \mathrm{ft}$ upstream from mouth of DP Canyon wash and 2.4 mi upstream from NM State Highway 4.

DRAINAGE AREA. $8.58 \mathrm{mi}^{2}$.

PERIOD OF RECORD. July 1994 to September 30, 1995.

GAGE. Data logger with cellular telemetry and concrete control. Elevation of gage is $6,580 \mathrm{ft}$ above National Geodetic Vertical Datum of 1929, from topographic map.

REMARKS. Water discharge records fair. Flow partially regulated by Los Alamos Reservoir about 2.5 mi upstream.

EXTREMES OUTSIDE PERIOD OF RECORD. Flood of July 31, 1968, was 329 cfs from slope area determination. Gage height was established later at $3.71 \mathrm{ft}$ present datum.

EXTREMES FOR PERIOD OF RECORD. Maximum discharge 12 cfs, Oct. 14, 1994, gage height, $1.50 \mathrm{ft}$. No flow at times.

EXTREMES FOR CURRENT WATER YEAR. Maximum discharge 12 cfs at 2330 hrs Oct. 14, 1994, gage height $1.50 \mathrm{ft}$. No flow at times

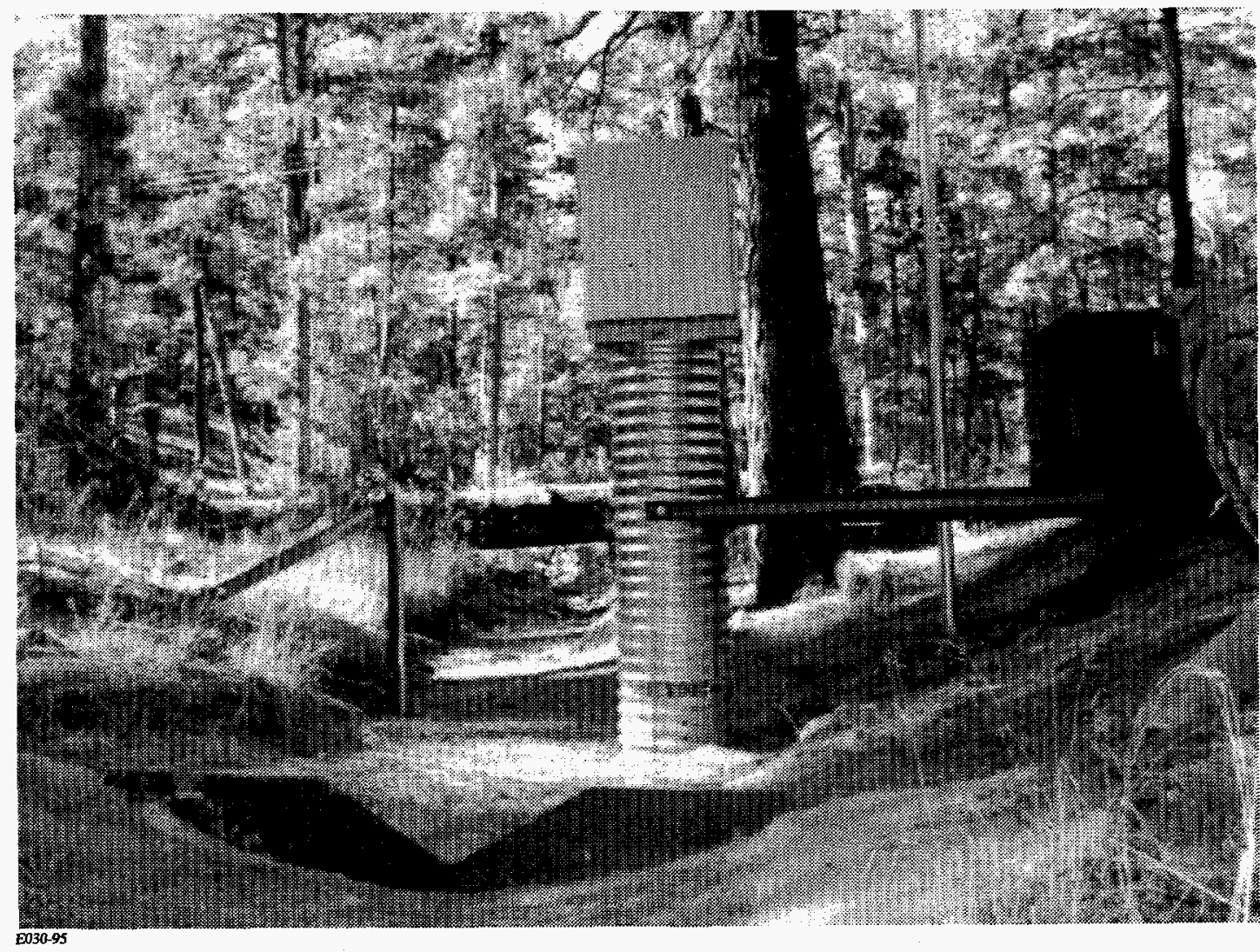


8313030 LOS ALAMOS CANYON BELOW TA-2 NEAR LOS ALAMOS, NM

DAILY DISCHARGE IN CUBIC FEET PER SECOND

WATER YEAR OCTOBER 1994 TO SEPTEMBER 1995

\begin{tabular}{|c|c|c|c|c|c|c|c|c|c|c|c|c|}
\hline Day & OCT & NOV & DEC & JAN & FEB & MAR & APR & MAY & JuN & JUL & AUG & SEP \\
\hline 1 & 0 & 0 & 0.82 & 0 & 0 & 0.01 & 0.62 & 5.5 & 1.4 & 0.17 & 0 & 0.01 \\
\hline 2 & 0 & 0 & 0.80 & 0 & 0 & 0.04 & 0.57 & 5.7 & 1.2 & 0 & 0 & 0 \\
\hline 3 & 0 & 0 & 0.80 & 0 & 0 & 0.09 & 0.49 & 6.2 & 1.2 & 0 & 0 & 0 \\
\hline 4 & 0 & 0 & 0.80 & 0 & 0 & 0.27 & 0.42 & 5.7 & 1.1 & 0 & 0 & 0 \\
\hline 5 & 0 & 0 & 0.80 & 0 & 0 & 0.49 & 0.34 & 5.6 & 0.73 & 0 & 0.49 & 0 \\
\hline 6 & 0 & 0 & 0.80 & 0 & 0 & 1.3 & 0.28 & 5.7 & 0.12 & 0 & 0 & 0.01 \\
\hline 7 & 0 & 0 & 0.50 & 0 & 0 & 1.6 & 0.38 & 5.4 & 0 & 0 & 0 & 0.65 \\
\hline 8 & 0 & 0 & 0.20 & 0 & 0 & 1.5 & 0.51 & 5.1 & 0 & 0 & 0 & 0.21 \\
\hline 9 & 0 & 0 & 0.08 & 0 & 0 & 1.4 & 0.64 & 4.4 & 0 & 0 & 0 & 0.69 \\
\hline 10 & 0 & 0 & 0.04 & 0 & 0 & 1.1 & 0.57 & 4.0 & 0 & 0 & 0 & 0.27 \\
\hline 11 & 0 & 0 & 0.01 & 0 & 0 & 0.97 & 0.86 & 4.3 & 0 & 0 & 0.33 & 0.84 \\
\hline 12 & 0 & 7.1 & 0 & 0 & 0 & 0.91 & 0.75 & 4.7 & 0 & 0 & 0 & 0.23 \\
\hline 13 & 0 & 3.5 & 0 & 0 & 0 & 0.82 & 0.66 & 5.0 & 0 & 0 & 0 & 0.05 \\
\hline 14 & 0.96 & 3.1 & 0 & 0 & 0 & 0.77 & 0.65 & 5.0 & 0 & 0 & 0 & 0.01 \\
\hline 15 & 1.9 & 1.6 & 0 & 0 & 0 & 0.75 & 0.73 & 5.0 & 0 & 0 & 0 & 0 \\
\hline 16 & 0.72 & 0.65 & 0 & 0 & 0 & 0.79 & 0.82 & 5.1 & 0 & 0.09 & 0 & 0 \\
\hline 17 & 1.3 & 0.40 & 0 & 0 & 0 & 0.86 & 0.88 & 5.7 & 1.5 & 0 & 0 & 0 \\
\hline 18 & 0 & 0.33 & 0 & 0 & 0 & 1.0 & 0.98 & 5.7 & 0.64 & 1.0 & 0 & 0 \\
\hline 19 & 0 & 0.31 & 0 & 0 & 0 & 1.3 & 0.90 & 5.0 & 0.36 & 0.03 & 0 & 0 \\
\hline 20 & 0 & 0.27 & 0 & 0 & 0 & 1.6 & 0.83 & 4.4 & 0.20 & 0 & 0 & 0 \\
\hline 21 & 0 & 0.26 & 0 & 0 & 0 & 1.8 & 0.34 & 3.7 & 0.09 & 0 & 0 & 0 \\
\hline 22 & 0 & 0.24 & 0 & 0 & 0 & 2.2 & 0.74 & 3.5 & 0.05 & 0 & 0 & 0 \\
\hline 23 & 0 & 0.23 & 0 & 0 & 0 & 2.2 & 0.66 & 3.7 & 0.01 & 0 & 0 & 0 \\
\hline 24 & 0 & 0.22 & 0 & 0 & 0 & 2.0 & 0.70 & 3.5 & 0 & 0 & 0.16 & 0 \\
\hline 25 & 0 & 0.20 & 0 & 0 & 0 & 1.8 & 1.2 & 2.5 & 0.09 & 0 & 0.15 & 0 \\
\hline 26 & 0 & 0.20 & 0 & 0 & 0 & 1.5 & 1.1 & 2.1 & 0.63 & 0 & 0.84 & 0 \\
\hline 27 & 0 & 0.30 & 0 & 0 & 0 & 1.3 & 1.1 & 1.8 & 0.33 & 0 & 0.91 & 0 \\
\hline 28 & 0 & 0.55 & 0 & 0 & 0 & 1.2 & 1.7 & 1.8 & 0.26 & 0 & 0.27 & 0 \\
\hline 29 & 0 & 0.80 & 0 & 0 & - & 0.96 & 2.9 & 4.4 & 0.11 & 0 & 0.68 & 0 \\
\hline 30 & 0 & 0.81 & 0 & 0 & - & 0.78 & 4.8 & 2.3 & 0.23 & 0 & 0.37 & 0 \\
\hline 31 & 0 & $\ldots$ & 0 & 0 & - & 0.66 & - & 1.6 & - & 0 & 0.13 & 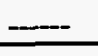 \\
\hline Total & 4.88 & 21.07 & 5.65 & 0 & 0 & 33.97 & 28.12 & 134.1 & 10.25 & 1.29 & 4.33 & 2.97 \\
\hline Mean & 0.16 & 0.7 & 0.18 & 0 & 0 & 1.1 & & 4.33 & 0.34 & 0.042 & 0.14 & 0.099 \\
\hline & & & & & & & 0.94 & & & & & \\
\hline Max & 1.9 & 7.1 & 0.82 & 0 & 0 & 2.2 & 4.8 & 6.2 & 1.5 & 1 & 0.91 & 0.84 \\
\hline Min & 0 & 0 & 0 & 0 & 0 & 0.01 & 0.28 & 1.6 & 0 & 0 & 0 & 0 \\
\hline AC-FT & 9.7 & 42 & 11 & 0 & 0 & 67 & 56 & 266 & 20 & 2.6 & 8.6 & 5.9 \\
\hline
\end{tabular}

CY 1994: TOTAL* 31.60 MEAN 0.34 MAX 7.1 MIN 0 AC-FT 63

WATER YEAR 1995: TOTAL 246.63 MEAN 0.68 MAX 7.1 MIN 0 AC-FT 489

* Incomplete Record 


\section{Los Alamos CANYON NEAR Los Alamos, NM}

LOCATION. Lat $35^{\circ} 52^{\prime} 01^{\prime \prime}$, long $106^{\circ} 13^{\prime 2} 1^{\prime \prime}$, in SW1/4 sec. 20, T.19 M. R.7 E, Santa Fe County, on right bank, 1/4 mi upstream from NM State Highway 4, $2.7 \mathrm{mi}$ NW of White Rock, $\mathrm{NM}, 3.9 \mathrm{mi}$ E of Los Alamos, and $13.5 \mathrm{mi} \mathrm{SW}$ of Espanola.

DRAINAGE AREA. $9.08 \mathrm{mi}^{2}$.

PERIOD OF RECORD. November 1970 to June 1971, October 1991 to September 30, 1995.

GAGE. Data logger with cellular telemetry and concrete control. Elevation of gage is $6,380 \mathrm{ft}$ above National Geodetic Vertical Datum of 1929, from topographic map.

COOPERATION. Station operated by US Geological Survey through the 1995 water year.

REMARKS. Records fair. No diversion above station.

EXTREMES FOR PERIOD OF RECORD. Maximum discharge $137 \mathrm{ft}^{3} / \mathrm{s}$, Aug. 27, 1993, gage height $2.7 \mathrm{ft}$. No flow at times.

EXTREMES FOR CURRENT WATER YEAR. Maximum discharge $54 \mathrm{ft}^{3} / \mathrm{s}, 0025 \mathrm{hrs}$, Oct. 15, 1995, gage height $2.29 \mathrm{ft}$. No flow at times.

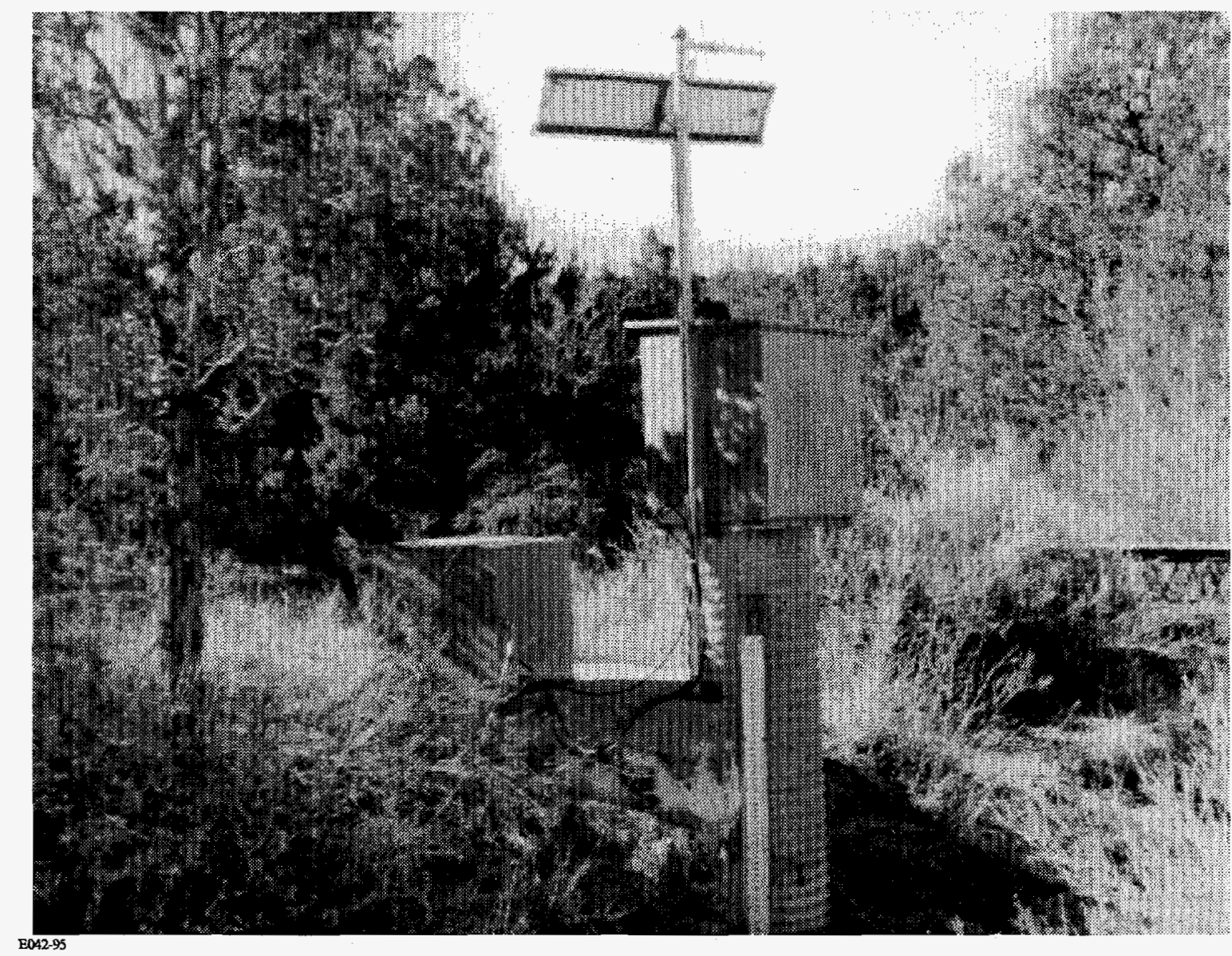


08313042 Los Alamos Canyon NEAR Los ALAMos, NM

DAILY DISCHARGE IN CUBIC FEET PER SECOND

WATER YEAR OCTOBER 1994 TO SEPTEMBER 1995

\begin{tabular}{|c|c|c|c|c|c|c|c|c|c|c|c|c|}
\hline Day & OCT & NOV & DEC & JAN & FEB & MAR & APR & MAY & JUN & JL & AUG & SEP \\
\hline 1 & 0 & 0 & 0 & 0 & 0 & 0 & 0.05 & 3.9 & 0.44 & 0.01 & 0 & 0 \\
\hline 2 & 0 & 0 & 0 & 0 & 0 & 0 & 0 & 4.1 & 0.42 & 0 & 0 & 0 \\
\hline 3 & 0 & 0 & 0 & 0 & 0 & 0 & 0 & 4.5 & 0.40 & 0 & 0 & 0 \\
\hline 4 & 0 & 0 & 0 & 0 & 0 & 0 & 0 & 4.4 & 0.36 & 0 & 0 & 0 \\
\hline 5 & 0 & 0 & 0 & 0 & 0 & 0 & 0 & 5.3 & 0.33 & 0 & 0 & 0 \\
\hline 6 & 0 & 0 & 0 & 0 & 0 & 0 & 0 & 4.2 & 0.30 & 0 & 0 & 0 \\
\hline 7 & 0 & 0 & 0 & 0 & 0 & 0 & 0 & 4.1 & 0.27 & 0 & 0 & 1.1 \\
\hline 8 & 0 & 0 & 0 & 0 & 0 & 0 & 0 & 4.0 & 0.20 & 0 & 0 & 0.38 \\
\hline 9 & 0 & 0 & 0 & 0 & 0 & 0 & 0 & 3.6 & 0.15 & 0 & 0 & 0.21 \\
\hline 10 & 0 & 0 & 0 & 0 & 0 & 0 & 0.12 & 3.2 & 0.13 & 0 & 0 & 0.05 \\
\hline 11 & 0 & 0 & 0 & 0 & 0 & 0 & 0.23 & 3.6 & 0.08 & 0 & 0 & 0.54 \\
\hline 12 & 0 & 9.5 & 0 & 0 & 0 & 0 & 0.05 & 3.9 & 0.05 & 0 & 0 & 0 \\
\hline 13 & 0 & 0.05 & 0 & 0 & 0 & 0 & 0 & 4.5 & 0.01 & 0 & 0 & 0 \\
\hline 14 & 1.3 & 0.68 & 0 & 0 & 0.10 & 0 & 0 & 4.5 & 0.03 & 0 & 0 & 0 \\
\hline 15 & 6.9 & 0.80 & 0 & 0 & 0.28 & 0 & 0.02 & 4.5 & 0.02 & 0 & 0 & 0 \\
\hline 16 & 0.97 & 0.44 & 0 & 0 & 0.02 & 0 & 0.05 & 4.7 & 0.01 & 0.20 & 0 & 0 \\
\hline 17 & 2.1 & 0.20 & 0 & 0 & 0 & 0 & 0.12 & 5.5 & 1.1 & 0 & 0 & 0 \\
\hline 18 & 0 & 1.1 & 0 & 0 & 0 & 0 & 0.15 & 5.7 & 0.27 & 2.4 & 0 & 0 \\
\hline 19 & 0 & 0 & 0 & 0 & 0 & 0 & 0.15 & 4.6 & 0 & 0.40 & 0 & 0 \\
\hline 20 & 0 & 0 & 0 & 0 & 0 & 0 & 0.13 & 4.1 & 0 & 0 & 0 & 0 \\
\hline 21 & 0 & 0 & 0 & 0 & 0 & 0 & 0.11 & 3.4 & 0 & 0 & 0 & 0 \\
\hline 22 & 0 & 0 & 0 & 0 & 0 & 0.42 & 0.34 & 3.1 & 0 & 0 & 0 & 0 \\
\hline 23 & 0 & 0 & 0 & 0 & 0 & 0.87 & 0.29 & 3.2 & 0 & 0 & 0 & 0 \\
\hline 24 & 0 & 0 & 0 & 0 & 0 & 0.85 & 0.29 & 3.1 & 0 & 0 & 0 & 0 \\
\hline 25 & 0 & 0 & 0 & 0 & 0 & 0.58 & 0.21 & 2.4 & 0.06 & 0 & 0 & 0 \\
\hline 26 & 0 & 0 & 0 & 0 & 0 & 0.83 & 0.27 & 1.8 & 0.99 & 0 & 0.41 & 0 \\
\hline 27 & 0 & 0 & 0 & 0 & 0 & 1.1 & 0.39 & 1.6 & 0.01 & 0 & 0.04 & 0 \\
\hline 28 & 0 & 0 & 0 & 0 & 0 & 0.24 & 0.65 & 1.3 & 0.05 & 0 & 0 & 0 \\
\hline 29 & 0 & 0 & 0 & 0 & 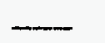 & 0.20 & 1.1 & 8.20 & 0 & 0 & 0.31 & 0 \\
\hline 30 & 0 & 0 & 0 & 0 & - & 0.12 & 2.9 & 0.82 & 0.06 & 0 & 0.07 & 0 \\
\hline 31 & 0 & - & 0 & 0 & - & 0.09 & - & 0.45 & - & 0 & 0 & - \\
\hline Total & 11.27 & 12.77 & 0 & 0 & 0.4 & 5.3 & 7.62 & 116.27 & 5.74 & 3.01 & 0.83 & 2.28 \\
\hline Mean & 0.36 & 0.43 & 0 & 0 & 0.014 & 0.17 & 0.25 & 3.75 & 0.19 & 0.097 & 0.027 & 0.076 \\
\hline $\operatorname{Max}$ & 6.9 & 9.5 & 0 & 0 & 0.28 & 1.1 & 2.9 & 8.2 & 1.1 & 2.4 & 0.41 & 1.1 \\
\hline Min & 0 & 0 & 0 & 0 & 0 & 0 & 0 & 0.45 & 0 & 0 & 0 & 0 \\
\hline $\begin{array}{l}\text { AC- } \\
\text { FT }\end{array}$ & 22 & 25 & 0 & 0 & 0.8 & 11 & 15 & 231 & 11 & 6 & 1.6 & 4.5 \\
\hline
\end{tabular}

CY 1994: TOTAL* 24.04 MEAN 0.26 MAX 9.5 MIN 0 AC-FT 48

WATER YEAR 1995: TOTAL 165.49 MEAN 0.45 MAX 9.5 MIN 0 AC-FT 328

* Incomplete Record 



\section{PUeblo Canyon near Los Alamos, NM}

DAILY DISCHARGE IN CUBIC FEET PER SECOND

WATER YEAR OCTOBER 1994 TO SEPTEMBER 1995

\begin{tabular}{|c|c|c|c|c|c|c|c|c|c|c|c|c|}
\hline Day & OCT & NOV & DEC & JAN & FEB & MAR & APR & MAY & JUN & JUL & AUG & SEP \\
\hline 1 & 0.89 & 0.66 & 0.84 & 1.2 & 1.0 & 1.4 & 1.5 & 0.79 & 1.3 & 1.7 & 2.4 & 2.2 \\
\hline 2 & 1.1 & 0.89 & 0.90 & 1.3 & 1.1 & 1.5 & 1.6 & 0.89 & 0.62 & 1.5 & 0.97 & 1.7 \\
\hline 3 & 1.0 & 0.95 & 0.77 & 1.5 & 1.1 & 1.4 & 1.5 & 0.84 & 0.82 & 1.6 & 0.84 & 0.97 \\
\hline 4 & 1.0 & 1.0 & 0.85 & 1.4 & 1.1 & 1.5 & 1.6 & 0.88 & 0.58 & 1.3 & 1.1 & 1.4 \\
\hline 5 & 1.4 & 1.2 & 0.89 & 1.2 & 1.2 & 1.5 & 1.3 & 0.81 & 0.67 & 1.1 & 1.7 & 1.3 \\
\hline 6 & 1.2 & 1.3 & 1.1 & 1.4 & 1.2 & 1.6 & 1.3 & 0.92 & 0.60 & 0.70 & 2.1 & 1.0 \\
\hline 7 & 1.0 & 0.99 & 1.3 & 1.3 & 1.3 & 1.5 & 1.4 & 0.83 & 0.70 & 1.0 & 1.3 & 1.9 \\
\hline 8 & 1.4 & 1.1 & 1.3 & 1.0 & 1.2 & 1.4 & 1.5 & 0.87 & 0.69 & 0.81 & 1.1 & 2.1 \\
\hline 9 & 1.4 & 1.1 & 1.3 & 1.1 & 1.1 & 1.4 & 1.1 & 0.77 & 0.78 & 1.1 & 1.3 & 2.2 \\
\hline 10 & 1.4 & 1.1 & 1.2 & 1.1 & 1.1 & 1.1 & 1.6 & 0.58 & 0.58 & 0.77 & 1.2 & 2.3 \\
\hline 11 & 1.1 & 1.1 & 1.2 & 1.0 & 1.1 & 1.3 & 1.6 & 0.89 & 0.73 & 0.96 & 1.5 & 1.7 \\
\hline 12 & 0.80 & 3.1 & 1.3 & 1.0 & 0.97 & 1.2 & 1.6 & 0.73 & 0.58 & 0.9 & 1.4 & 1.6 \\
\hline 13 & 0.48 & 1.5 & 1.2 & 0.97 & 1.0 & 1.4 & 1.6 & 0.53 & 0.65 & 2.0 & 1.4 & 1.5 \\
\hline 14 & 1.2 & 1.3 & 1.2 & 1.0 & 1.0 & 1.1 & 1.3 & 0.67 & 0.73 & 1.2 & 1.4 & 1.5 \\
\hline 15 & 1.9 & 0.98 & 1.3 & 1.0 & 1.1 & 1.3 & 1.2 & 0.53 & 0.79 & 1.1 & 1.9 & 0.68 \\
\hline 16 & 1.0 & 0.9 & 1.2 & 1.0 & 1.1 & 1.5 & 1.4 & 0.58 & 0.81 & 1.8 & 1.1 & 0.89 \\
\hline 17 & 3.1 & 0.88 & 1.3 & 1.0 & 1.1 & 1.3 & 1.4 & 0.65 & 1.4 & 1.6 & 1.1 & 1.5 \\
\hline 18 & 1.4 & 0.87 & 1.2 & 1.0 & 1.2 & 1.3 & 1.3 & 0.83 & 1.8 & 2.5 & 1.7 & 1.2 \\
\hline 19 & 0.92 & 0.94 & 1.2 & 0.97 & 1.2 & 1.2 & 1.4 & 0.96 & 1.6 & 2.5 & 1.5 & 1.4 \\
\hline 20 & 0.94 & 0.92 & 1.2 & 0.8 & 1.2 & 1.3 & 1.3 & 0.99 & 1.6 & 2.2 & 1.4 & 1.5 \\
\hline 21 & 0.62 & 0.92 & 1.1 & 0.89 & 1.3 & 1.1 & 1.4 & 0.73 & 1.0 & 1.8 & 1.5 & 1.1 \\
\hline 22 & 0.70 & 0.89 & 1.0 & 0.97 & 1.2 & 1.4 & 1.5 & 0.75 & 0.89 & 2.2 & 1.4 & 0.76 \\
\hline 23 & 0.76 & 0.91 & 1.1 & 0.97 & 1.2 & 1.2 & 1.5 & 0.47 & 0.85 & 2.4 & 0.76 & 1.6 \\
\hline 24 & 0.87 & 0.86 & 1.2 & 0.97 & 1.2 & 1.4 & 1.6 & 0.67 & 1.0 & 1.4 & 1.5 & 1.7 \\
\hline 25 & 0.72 & 0.82 & 1.1 & 1.0 & 1.3 & 1.3 & 1.6 & 0.77 & 0.84 & 0.89 & 1.7 & 1.9 \\
\hline 26 & 0.91 & 0.87 & 1.1 & 0.97 & 1.3 & 1.4 & 1.6 & 0.77 & 1.9 & 0.76 & 2.2 & 0.60 \\
\hline 27 & 0.78 & 0.87 & 1.2 & 0.93 & 1.4 & 1.5 & 1.5 & 0.72 & 1.7 & 0.76 & 2.5 & 1.1 \\
\hline 28 & 0.75 & 0.92 & 1.2 & 0.97 & 1.3 & 1.4 & 1.4 & 0.76 & 1.6 & 0.68 & 2.2 & 0.49 \\
\hline 29 & 0.97 & 0.87 & 1.2 & 0.97 & & 1.4 & 1.2 & 1.4 & 1.9 & 0.72 & 2.3 & 1.4 \\
\hline 30 & 0.99 & 0.84 & 1.2 & 0.97 & - & 1.5 & 0.87 & 1.4 & 1.6 & 0.76 & 2.5 & 1.5 \\
\hline 31 & 0.83 & - & 1.3 & 1.0 & - & 1.5 & - & 1.2 & - & 0.8 & 2.3 & - \\
\hline Total & 33.53 & 31.55 & 35.45 & 32.85 & 32.57 & 42.3 & 42.67 & 25.18 & 31.31 & 41.51 & 49.27 & 42.69 \\
\hline Mean & 1.08 & 1.05 & 1.14 & 1.06 & 1.16 & 1.36 & 1.42 & 0.81 & 1.04 & 1.34 & 1.59 & 1.42 \\
\hline Max & 3.1 & 3.1 & 1.3 & 1.5 & 1.4 & 1.6 & 1.6 & 1.4 & 1.9 & 2.5 & 2.5 & 2.3 \\
\hline Min & 0.48 & 0.66 & 0.77 & 0.8 & 0.97 & 1.1 & 0.87 & 0.47 & 0.58 & 0.68 & 0.76 & 0.49 \\
\hline $\begin{array}{l}\text { AC- } \\
\text { FT }\end{array}$ & 67 & 63 & 70 & 65 & 65 & 84 & 85 & 50 & 62 & 82 & 98 & 85 \\
\hline
\end{tabular}

CY 1994: TOTAL* 100.53 MEAN 1.09 MAX 3.1 MIN 0.48 AC-FT 199

WATER YEAR 1995: TOTAL 440.88 MEAN 1.21 MAX 3.1 MIN 0.47 AC-FT 874

* Incomplete Record 


\section{Sandia Canyon above HighWay 4 NEAR White Rock, NM}

LOCATION Lat $35^{\circ}$ 51'32", long $106^{\circ} 13^{\prime} 33^{\prime \prime}$, SE1/4SW1/4 sec. 20, T. 19 N, R.7 E, Santa Fe County, $0.25 \mathrm{mi}$ N of East Jemez Road and $0.5 \mathrm{mi}$ upstream from NM State Highway 4.

DRAINAGE AREA. $2.52 \mathrm{mi}^{2}$.

PERIOD OF RECORD. October 1993 to September 30, 1995.

GAGE. Data logger with cellular telemetry and concrete control. Elevation of gage is $6,495 \mathrm{ft}$. above National Geodetic Vertical Datum of 1929, from topographic map.

REMARKS. Water discharge records fair. Flow partially regulated by Los Almos Reservoir about 2.5 miles upstream.

EXTREMES FOR PERIOD OF RECORD. Maximum discharge 13 cfs Sept. 8, 1995, gage height $1.82 \mathrm{ft}$. No flow most of time.

EXTREMES FOR CURRENT WATER YEAR. Maximum discharge $13 \mathrm{cfs}$ at $1245 \mathrm{hrs}$ Sept. 8, 1995, gage height $1.82 \mathrm{ft}$. No flow most of time.

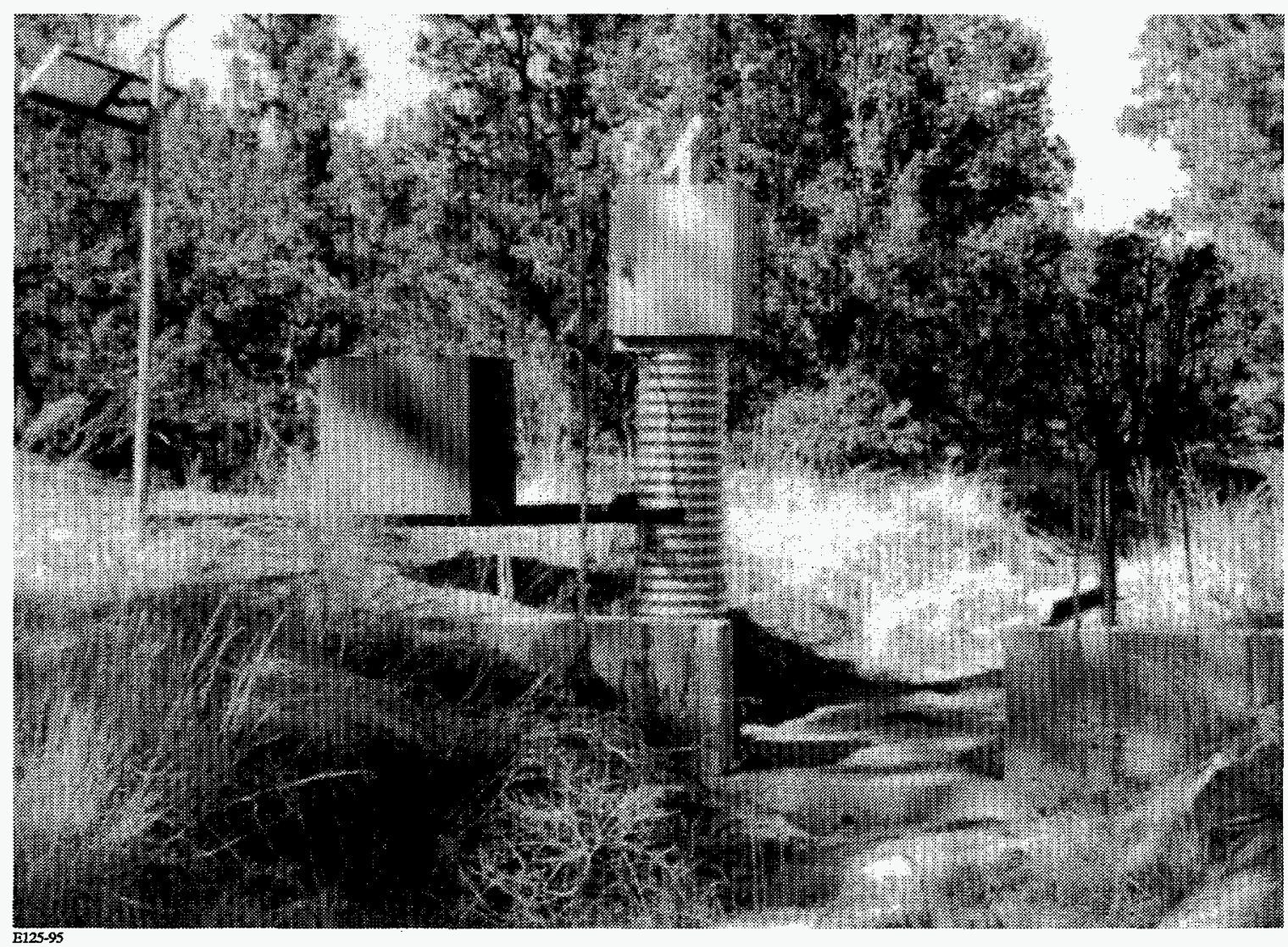


DAILY DISCHARGE IN CUBIC FEET PER SECOND

WATER YEAR OCTOBER 1994 TO SEPTEMBER 1995

\begin{tabular}{|c|c|c|c|c|c|c|c|c|c|c|c|c|}
\hline Day & OCT & NOV & DEC & JAN & FEB & MAR & APR & MAY & JUN & JUL & AUG & SEP \\
\hline 1 & 0 & 0 & 0 & 0 & 0 & 0 & 0 & 0 & 0 & 0 & 0 & 0 \\
\hline 2 & 0 & 0 & 0 & 0 & 0 & 0 & 0 & 0 & 0 & 0 & 0 & 0 \\
\hline 3 & 0 & 0 & 0 & 0 & 0 & 0 & 0 & 0 & 0 & 0 & 0 & 0 \\
\hline 4 & 0 & 0 & 0 & 0 & 0 & 0 & 0 & 0 & 0 & 0 & 0 & 0 \\
\hline 5 & 0 & 0 & 0 & 0 & 0 & 0 & 0 & 0 & 0 & 0 & 0 & 0 \\
\hline 6 & 0 & 0 & 0 & 0 & 0 & 0 & 0 & 0 & 0 & 0 & 0 & 0 \\
\hline 7 & 0 & 0 & 0 & 0 & 0 & 0 & 0 & 0 & 0 & 0 & 0 & 0 \\
\hline 8 & 0 & 0 & 0 & 0 & 0 & 0 & 0 & 0 & 0 & 0 & 0 & 1.4 \\
\hline 9 & 0 & 0 & 0 & 0 & 0 & 0 & 0 & 0 & 0 & 0 & 0 & 0 \\
\hline 10 & 0 & 0 & 0 & 0 & 0 & 0 & 0 & 0 & 0 & 0 & 0 & 0 \\
\hline 11 & 0 & 0.06 & 0 & 0 & 0 & 0 & 0 & 0 & 0 & 0 & 0.05 & 0 \\
\hline 12 & 0 & 0.40 & 0 & 0 & 0 & 0 & 0 & 0 & 0 & 0 & 0 & 0 \\
\hline 13 & 0 & 0 & 0 & 0 & 0 & 0 & 0 & 0 & 0 & 0 & 0.60 & 0 \\
\hline 14 & 0 & 0 & 0 & 0 & 0 & 0 & 0 & 0 & 0 & 0 & 0 & 0 \\
\hline 15 & 0 & 0 & 0 & 0 & 0 & 0 & 0 & 0 & 0 & 0 & 0 & 0 \\
\hline 16 & 0 & 0 & 0 & 0 & 0 & 0 & 0 & 0 & 0 & 0 & 0 & 0 \\
\hline 17 & 0 & 0 & 0 & 0 & 0 & 0 & 0 & 0 & 0 & 0 & 0 & 0 \\
\hline 18 & 0 & 0 & 0 & 0 & 0 & 0 & 0 & 0 & 0 & 0 & 0 & 0 \\
\hline 19 & 0 & 0 & 0 & 0 & 0 & 0 & 0 & 0 & 0 & 0 & 0 & 0 \\
\hline 20 & 0 & 0 & 0 & 0 & 0 & 0 & 0 & 0 & 0 & 0 & 0 & 0 \\
\hline 21 & 0 & 0 & 0 & 0 & 0 & 0 & 0 & 0 & 0 & 0 & 0 & 0 \\
\hline 22 & 0 & 0 & 0 & 0 & 0 & 0 & 0 & 0 & 0 & 0 & 0 & 0 \\
\hline 23 & 0 & 0 & 0 & 0 & 0 & 0 & 0 & 0 & 0 & 0 & 0 & 0 \\
\hline 24 & 0 & 0 & 0 & 0 & 0 & 0 & 0 & 0 & 0 & 0 & 0 & 0 \\
\hline 25 & 0 & 0 & 0 & 0 & 0 & 0 & 0 & 0 & 0 & 0 & 0 & 0 \\
\hline 26 & 0 & 0 & 0 & 0 & 0 & 0 & 0 & 0 & 0 & 0 & 0 & 0 \\
\hline 27 & 0 & 0 & 0 & 0 & 0 & 0 & 0 & 0 & 0 & 0 & 0 & 0 \\
\hline 28 & 0 & 0 & 0 & 0 & 0 & 0 & 0 & 0 & 0 & 0 & 0 & 0 \\
\hline 29 & 0 & 0 & 0 & 0 & - & 0 & 0 & 0 & 0 & 0 & 0 & 0 \\
\hline 30 & 0 & 0 & 0 & 0 & - & 0 & 0 & 0 & 0 & 0 & 0 & 0 \\
\hline 31 & 0 & - & 0 & 0 & - & 0 & - & 0 & -- & 0 & 0 & $=$ \\
\hline Total & 0 & 0.46 & 0 & 0 & 0 & 0 & 0 & 0 & 0 & 0 & 0.65 & 1.4 \\
\hline Mean & 0 & 0.015 & 0 & 0 & 0 & 0 & 0 & 0 & 0 & 0 & 0.021 & 0.047 \\
\hline $\operatorname{Max}$ & 0 & 0.4 & 0 & 0 & 0 & 0 & 0 & 0 & 0 & 0 & 0.6 & 1.4 \\
\hline Min & 0 & 0 & 0 & 0 & 0 & 0 & 0 & 0 & 0 & 0 & 0 & 0 \\
\hline AC-FT & 0 & 0.9 & 0 & 0 & 0 & 0 & 0 & 0 & 0 & 0 & 1.3 & 2.8 \\
\hline
\end{tabular}

CY 1994: TOTAL* 0.46 MEAN 0.005 MAX 0.4 MIN 0 AC-FT 0.9

WATER YEAR 1995: TOTAL 2.51 MEAN 0.007 MAX 1.4 MIN 0 AC-FT 5

* Incomplete Record 


\section{MORTANDAD CANYON AT TA-50 NEAR LOS ALAMOS, NM}

LOCATION. Lat $35^{\circ} 51^{\prime} 55^{\prime \prime}$, long $106^{\circ} 17^{\prime} 42^{\prime \prime}$, SW 1/4NE $1 / 2$ sec. 22 , T. 19 N, R.6 E, Los Alamos County, 0.6 N of Pajarito Road and .25 mi N of LANL TA-50 and 1/4 mi below TA-50 outfall.

DRAINAGE AREA. $0.49 \mathrm{mi}^{2}$.

PERIOD OF RECORD. May 10, 1995, to September 30, 1995.

GAGE. Data logger with cellular telemetry and steel "fabricated" Parshall Flume as low-water control. Elevation of gage is $7,040 \mathrm{ft}$ above National Geodetic Vertical Datum of 1929 from topographic map.

REMARKS. Water discharge records fair. Flow is mostly effluent from LANL TA-50.

EXTREMES OUTSIDE PERIOD OF RECORD. Flow of 34 cfs occurred Aug. 19, 1970, gage height $3.07 \mathrm{ft}$ from old data files of US Geological Survey.

EXTREMES FOR CURRENT WATER YEAR. Maximum discharge 9.7 cfs at 1525 hrs May 29, gage height $1.94 \mathrm{ft}$. No flow at times.

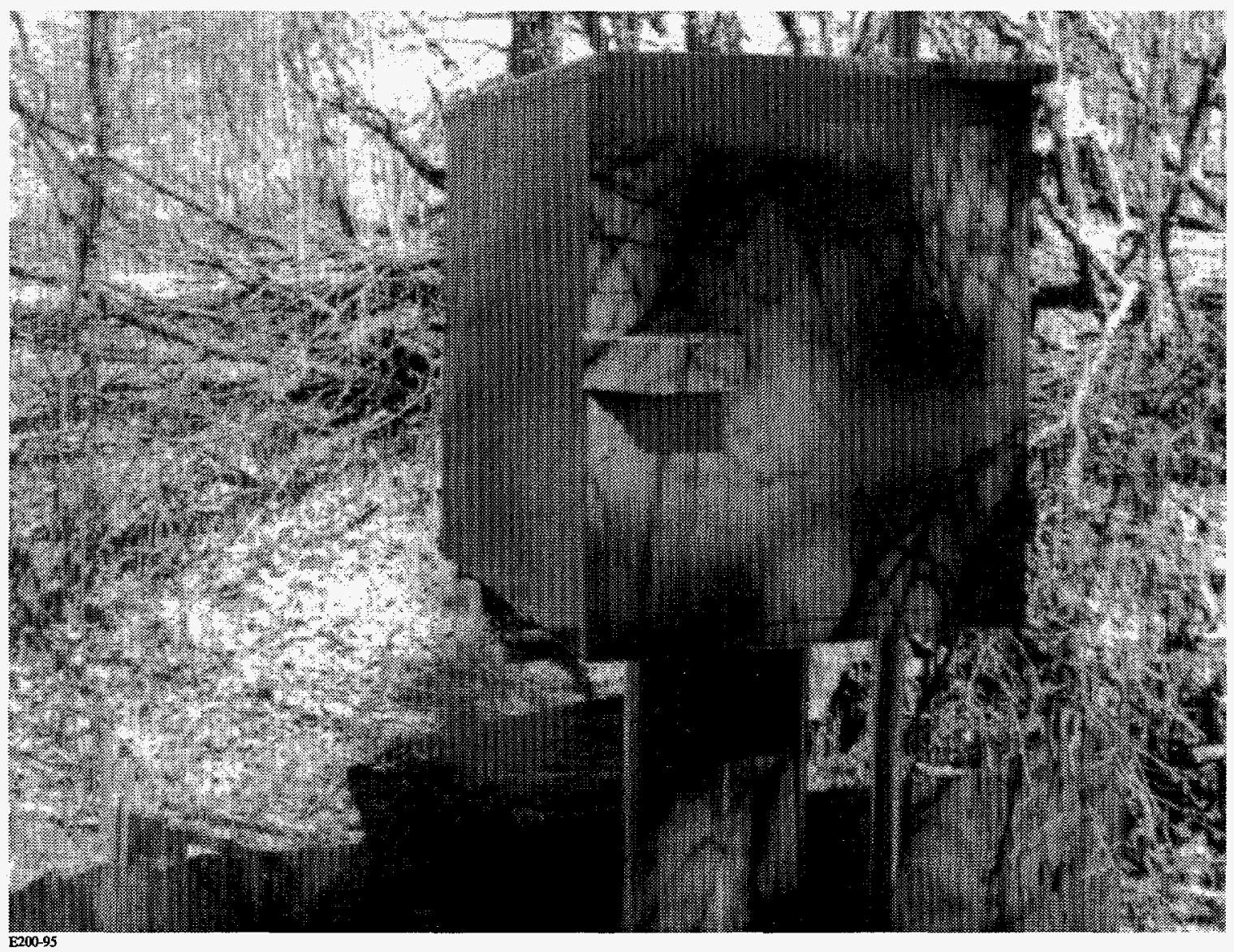




\section{MORTANDAD CANYON AT TA-50 NEAR LOS ALAMOS, NM}

DAILY DISCHARGE IN CUBIC FEET PER SECOND

WATER YEAR OCTOBER 1994 TO SEPTEMBER 1995

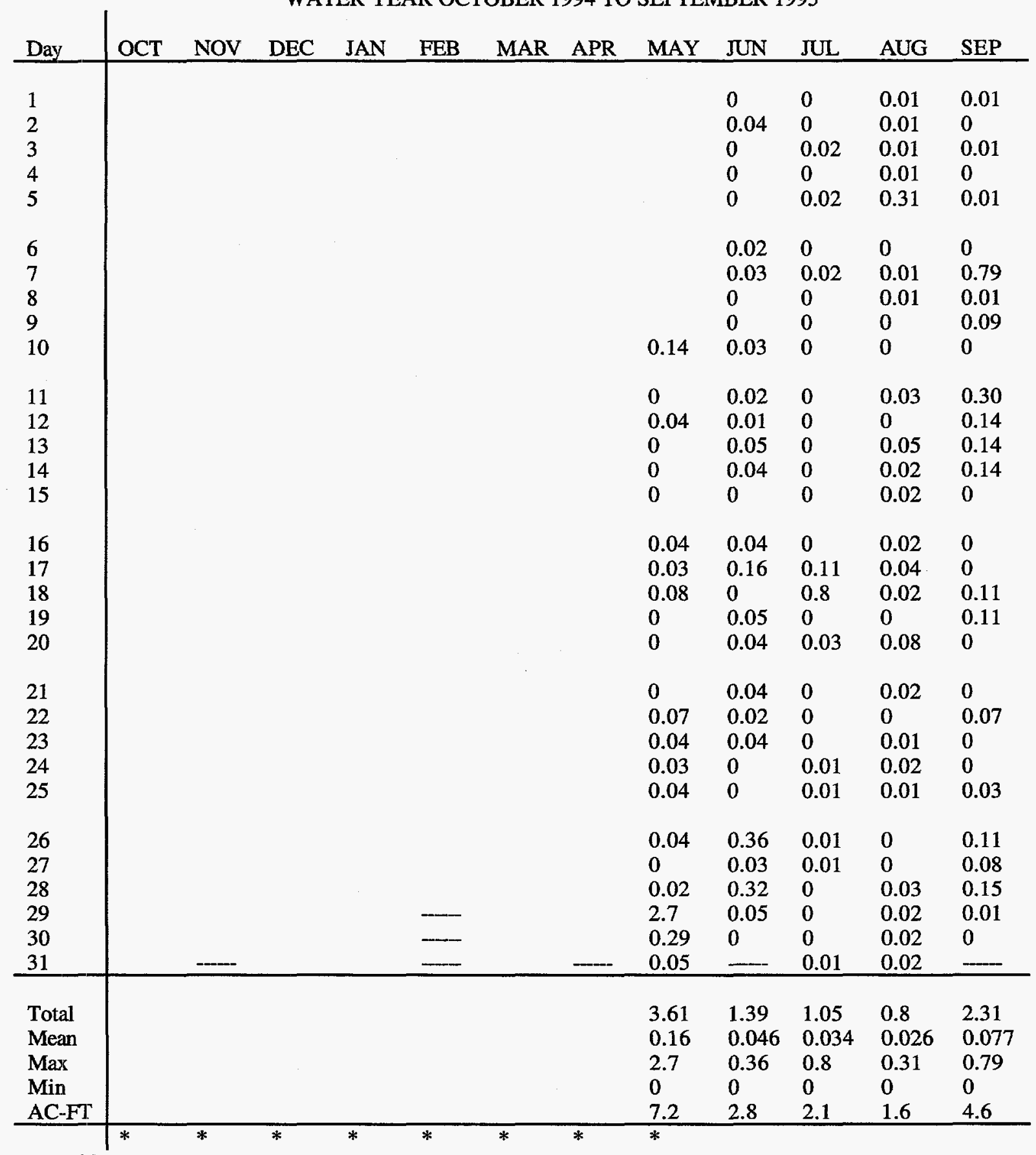

CY 1994: TOTAL* 0.0

WATIER YEAR 1995: TOTAL* 9.16 MEAN 0.064 MAX 2.7 MIN 0 AC-FT 18

* Incomplete Record 


\section{MORTANDAD CANYON AT LABORATORY BOUNDARY}

LOCATION. Lat $35^{\circ} 51^{\prime} 22^{\prime \prime}$, long $106^{\circ} 14^{\prime} 42^{\prime \prime}$, NW1/4 NW1/4 sec. 30, T. 19, R. 7 E., Santa Fe County, $100 \mathrm{ft}$ upstream from LANL/San Ildefonso Indian Reservation Boundary and $2.8 \mathrm{mi}$ upstream from NM State Highway 4.

DRAINAGE AREA.1.67 $\mathrm{mi}^{2}$.

PERIOD OF RECORD. October 1, 1993, to September 30, 1995.

GAGE. Data logger with cellular telemetry and concrete control. Elevation of gage is $6,665 \mathrm{ft}$. above National Geodetic Vertical Datum of 1929 from topographic map.

EXTREMES FOR PERIOD OF RECORD. No flow for period.

EXTREMES FOR CURRENT WATER YEAR. No flow for year.

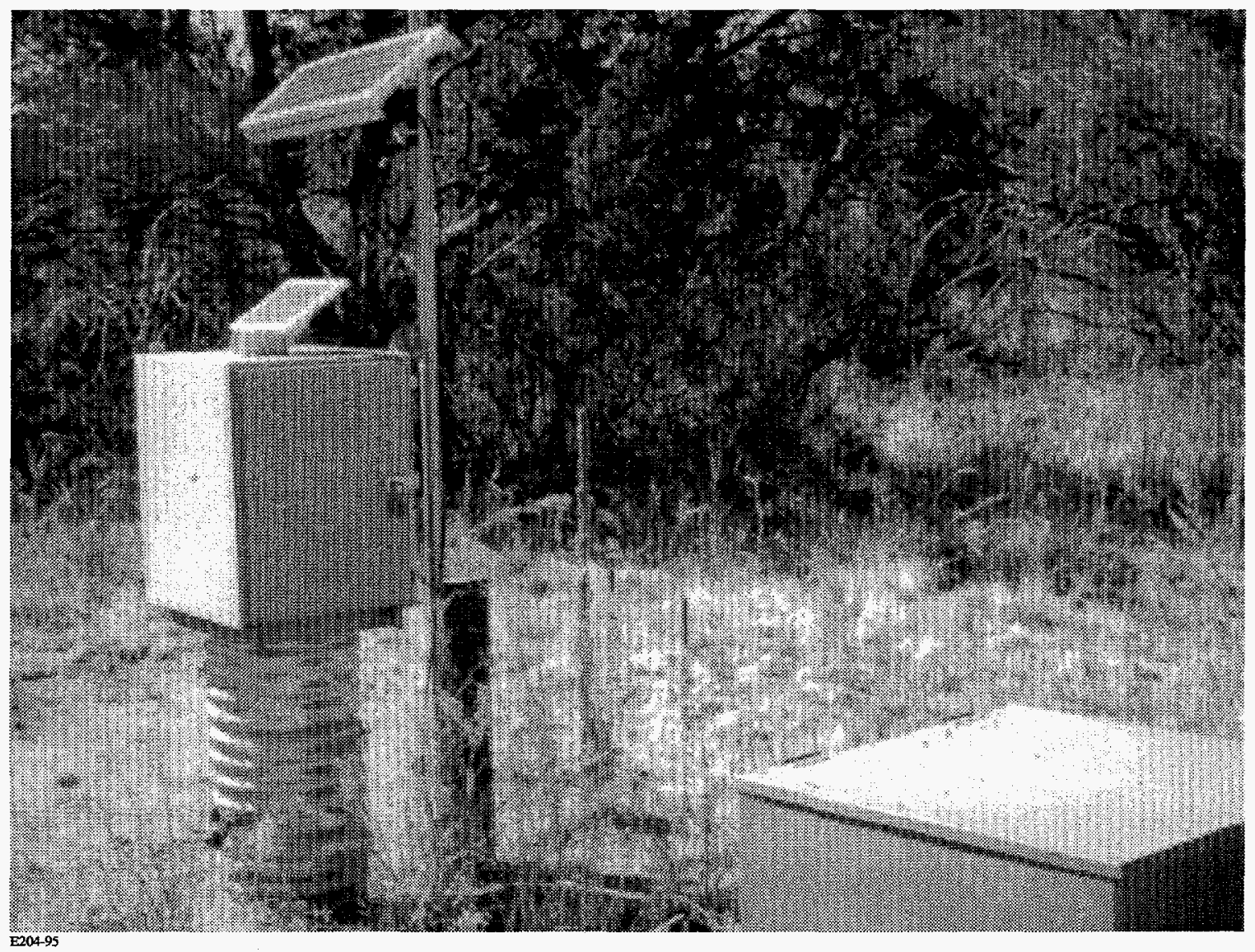


8313204 MORTANDAD CANYON AT LABORATORY BOUNDARY

DAILY DISCHARGE IN CUBIC FEET PER SECOND

WATER YEAR OCTOBER 1994 TO SEPTEMBER 1995

\begin{tabular}{|c|c|c|c|c|c|c|c|c|c|c|c|c|}
\hline Day & OCT & NOV & DEC & JAN & FEB & MAR & APR & MAY & JUN & JUL & AUG & SEP \\
\hline 1 & 0 & 0 & 0 & 0 & 0 & 0 & 0 & 0 & 0 & 0 & 0 & 0 \\
\hline 2 & 0 & 0 & 0 & 0 & 0 & 0 & 0 & 0 & 0 & 0 & 0 & 0 \\
\hline 3 & 0 & 0 & 0 & 0 & 0 & 0 & 0 & 0 & 0 & 0 & 0 & 0 \\
\hline 4 & 0 & 0 & 0 & 0 & 0 & 0 & 0 & 0 & 0 & 0 & 0 & 0 \\
\hline 5 & 0 & 0 & 0 & 0 & 0 & 0 & 0 & 0 & 0 & 0 & 0 & 0 \\
\hline 6 & 0 & 0 & 0 & 0 & 0 & 0 & 0 & 0 & 0 & 0 & 0 & 0 \\
\hline 7 & 0. & 0 & 0 & 0 & 0 & 0 & 0 & 0 & 0 & 0 & 0 & 0 \\
\hline 8 & 0 & 0 & 0 & 0 & 0 & 0 & 0 & 0 & 0 & 0 & 0 & 0 \\
\hline 9 & 0 & 0 & 0 & 0 & 0 & 0 & 0 & 0 & 0 & 0 & 0 & 0 \\
\hline 10 & 0 & 0 & 0 & 0 & 0 & 0 & 0 & 0 & 0 & 0 & 0 & 0 \\
\hline 11 & 0 & 0 & 0 & 0 & 0 & 0 & 0 & 0 & 0 & 0 & 0 & 0 \\
\hline 12 & 0 & 0 & 0 & 0 & 0 & 0 & 0 & 0 & 0 & 0 & 0 & 0 \\
\hline 13 & 0 & 0 & 0 & 0 & 0 & 0 & 0 & 0 & 0 & 0 & 0 & 0 \\
\hline 14 & 0 & 0 & 0 & 0 & 0 & 0 & 0 & 0 & 0 & 0 & 0 & 0 \\
\hline 15 & 0 & 0 & 0 & 0 & 0 & 0 & 0 & 0 & 0 & 0 & 0 & 0 \\
\hline 16 & 0 & 0 & 0 & 0 & 0 & 0 & 0 & 0 & 0 & 0 & 0 & 0 \\
\hline 17 & 0 & 0 & 0 & 0 & 0 & 0 & 0 & 0 & 0 & 0 & 0 & 0 \\
\hline 18 & 0 & 0 & 0 & 0 & 0 & 0 & 0 & 0 & 0 & 0 & 0 & 0 \\
\hline 19 & 0 & 0 & 0 & 0 & 0 & 0 & 0 & 0 & 0 & 0 & 0 & 0 \\
\hline 20 & 0 & 0 & 0 & 0 & 0 & 0 & 0 & 0 & 0 & 0 & 0 & 0 \\
\hline 21 & 0 & 0 & 0 & 0 & 0 & 0 & 0 & 0 & 0 & 0 & 0 & 0 \\
\hline 22 & 0 & 0 & 0 & 0 & 0 & 0 & 0 & 0 & 0 & 0 & 0 & 0 \\
\hline 23 & 0 & 0 & 0 & 0 & 0 & 0 & 0 & 0 & 0 & 0 & 0 & 0 \\
\hline 24 & 0 & 0 & 0 & 0 & 0 & 0 & 0 & 0 & 0 & 0 & 0 & 0 \\
\hline 25 & 0 & 0 & 0 & 0 & 0 & 0 & 0 & 0 & 0 & 0 & 0 & 0 \\
\hline 26 & 0 & 0 & 0 & 0 & 0 & 0 & 0 & 0 & 0 & 0 & 0 & 0 \\
\hline 27 & 0 & 0 & 0 & 0 & 0 & 0 & 0 & 0 & 0 & 0 & 0 & 0 \\
\hline 28 & 0 & 0 & 0 & 0 & 0 & 0 & 0 & 0 & 0 & 0 & 0 & 0 \\
\hline 29 & 0 & 0 & 0 & 0 & - & 0 & 0 & 0 & 0 & 0 & 0 & 0 \\
\hline 30 & 0 & 0 & 0 & 0 & - & 0 & 0 & 0 & 0 & 0 & 0 & 0 \\
\hline 31 & 0 & - & 0 & 0 & - & 0 & - & 0 & $\cdots$ & 0 & 0 & - \\
\hline Total & 0 & 0 & 0 & 0 & 0 & 0 & 0 & 0 & 0 & 0 & 0 & 0 \\
\hline Mean & 0 & 0 & 0 & 0 & 0 & 0 & 0 & 0 & 0 & 0 & 0 & 0 \\
\hline $\operatorname{Max}$ & 0 & 0 & 0 & 0 & 0 & 0 & 0 & 0 & 0 & 0 & 0 & 0 \\
\hline Min & 0 & 0 & 0 & 0 & 0 & 0 & 0 & 0 & 0 & 0 & 0 & 0 \\
\hline $\begin{array}{l}\text { AC- } \\
\text { FT } \\
\end{array}$ & 0 & 0 & 0 & 0 & 0 & 0 & 0 & 0 & 0 & 0 & 0 & 0 \\
\hline
\end{tabular}

CY 1994: TOTAL* 0 MEAN 0 MAX 0 MIN 0 AC-FT 0

WATER YEAR 1995: TOTAL 0 MEAN 0 MAX 0 MIN 0 AC-FT 0

* Incomplete Record 


\section{Cañada del Buey above White Rock, NM}

LOCATION. Lat $35^{\circ} 50^{\prime} 07^{\prime \prime}$, long $106^{\circ} 14^{\prime 2} 29^{\prime \prime}$, in Ramon Vigil Grant, Los Alamos County, 0.1 mi south of Santa Fe/Los Alamos County Line and 2.5 mi upstream from NM State Highway 4 in White Rock.

DRAINAGE AREA. $1.58 \mathrm{mi}^{2}$.

PERIOD OF RECORD. October 1993 to September 30, 1995.

GAGE. Data logger with cellular telemetry and concrete control. Elevation of gage is $6,620 \mathrm{ft}$ above National Geodetical Vertical Datum of 1929 from topographic map.

EXTREMES FOR PERIOD OF RECORD. Maximum discharge $17 \mathrm{cfs}$ Sept. 8, 1995, gage height $2.71 \mathrm{ft}$. No flow most of time.

EXTREMES FOR CURRENT WATER YEAR. Maximum discharge $17 \mathrm{cfs}$. at $1315 \mathrm{hrs}$ Sept. 8, 1995, gage height $2.71 \mathrm{ft}$. No flow most of the time.

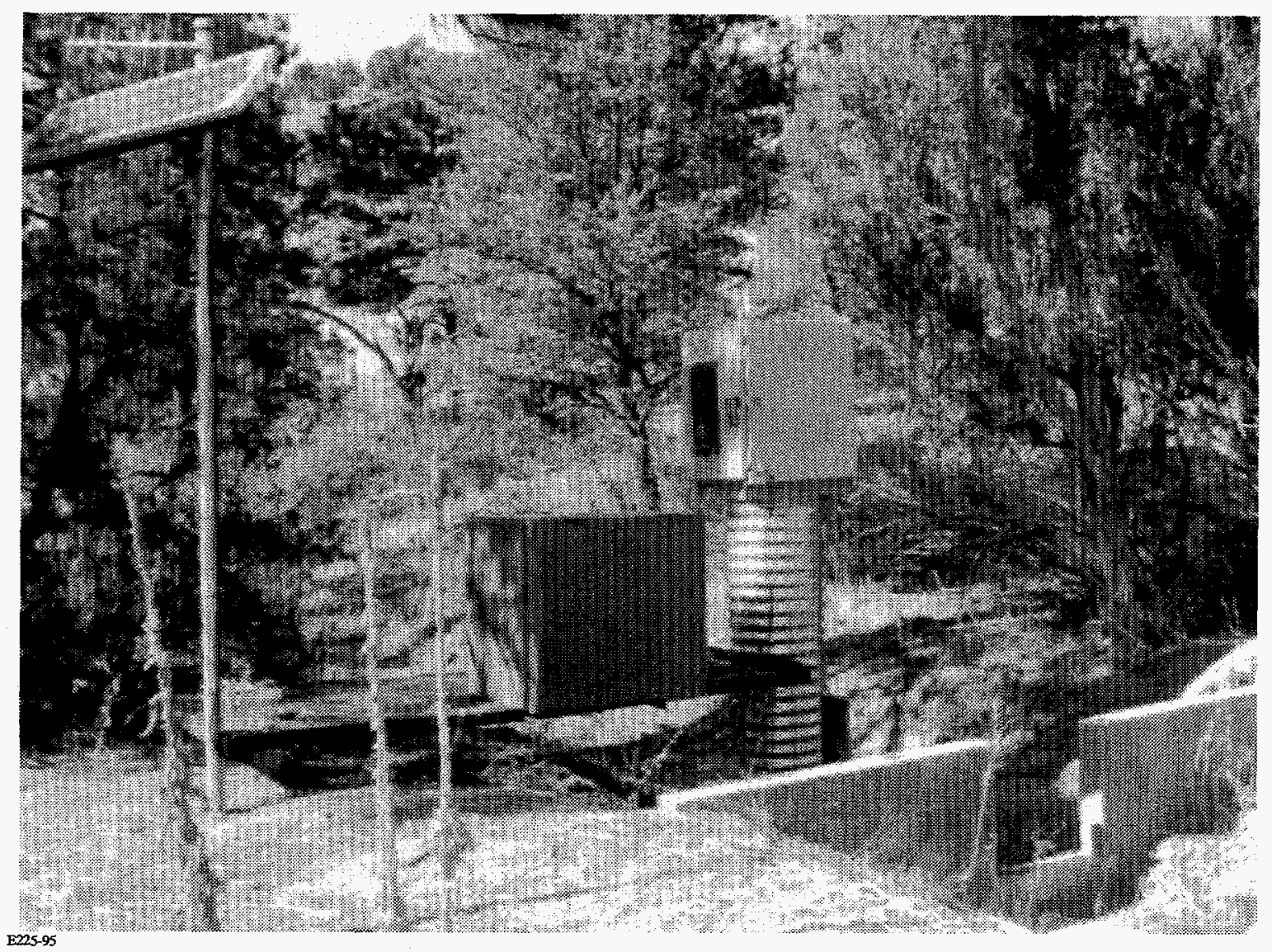


08313225 Cañada del Buey above White Rock, NM

DAILY DISCHARGE IN CUBIC FEET PER SECOND

WATER YEAR OCTOBER 1994 TO SEPTEMBER 1995

\begin{tabular}{|c|c|c|c|c|c|c|c|c|c|c|c|c|}
\hline Day & OCT & NOV & DEC & JAN & FEB & MAR & APR & MAY & JUN & JUL & AUG & SEP \\
\hline 1 & 0 & 0 & 0 & 0 & 0 & 0 & 0 & 0 & 0 & 0 & 0 & 0 \\
\hline 2 & 0 & 0 & 0 & 0 & 0 & 0 & 0 & 0 & 0 & 0 & 0 & 0 \\
\hline 3 & 0 & 0 & 0 & 0 & 0 & 0 & 0 & 0 & 0 & 0 & 0 & 0 \\
\hline 4 & 0 & 0 & 0 & 0 & 0 & 0 & 0 & 0 & 0 & 0 & 0 & 0 \\
\hline 5 & 0 & 0 & 0 & 0 & 0 & 0 & 0 & 0 & 0 & 0 & 0 & 0 \\
\hline 6 & 0 & 0 & 0 & 0 & 0 & 0 & 0 & 0 & 0 & 0 & 0 & 0 \\
\hline 7 & 0 & 0 & 0 & 0 & 0 & 0 & 0 & 0 & 0 & 0 & 0 & 0 \\
\hline 8 & 0 & 0 & 0 & 0 & 0 & 0 & 0 & 0 & 0 & 0 & 0 & 0.20 \\
\hline 9 & 0 & 0 & 0 & 0 & 0 & 0 & 0 & 0 & 0 & 0 & 0 & 0 \\
\hline 10 & 0 & 0 & 0 & 0 & 0 & 0 & 0 & 0 & 0 & 0 & 0 & 0 \\
\hline 11 & 0 & 0 & 0 & 0 & 0 & 0 & 0 & 0 & 0 & 0 & 0 & 0 \\
\hline 12 & 0 & 0 & 0 & 0 & 0 & 0 & 0 & 0 & 0 & 0 & 0 & 0 \\
\hline 13 & 0 & 0 & 0 & 0 & 0 & 0 & 0 & 0 & 0 & 0 & 0 & 0 \\
\hline 14 & 0 & 0 & 0 & 0 & 0 & 0 & 0 & 0 & 0 & 0 & 0 & 0 \\
\hline 15 & 0 & 0 & 0 & 0 & 0 & 0 & 0 & 0 & 0 & 0 & 0 & 0 \\
\hline 16 & 0 & 0 & 0 & 0 & 0 & 0 & 0 & 0 & 0 & 0 & 0 & 0 \\
\hline 17 & 0 & 0 & 0 & 0 & 0 & 0 & 0 & 0 & 0 & 0 & 0 & 0 \\
\hline 18 & 0 & 0 & 0 & 0 & 0 & 0 & 0 & 0 & 0 & 0 & 0 & 0 \\
\hline 19 & 0 & 0 & 0 & 0 & 0 & 0 & 0 & 0 & 0 & 0 & 0 & 0 \\
\hline 20 & 0 & 0 & 0 & 0 & 0 & 0 & 0 & 0 & 0 & 0 & 0 & 0 \\
\hline 21 & 0 & 0 & 0 & 0 & 0 & 0 & 0 & 0 & 0 & 0 & 0 & 0 \\
\hline 22 & 0 & 0 & 0 & 0 & 0 & 0 & 0 & 0 & 0 & 0 & 0 & 0 \\
\hline 23 & 0 & 0 & 0 & 0 & 0 & 0 & 0 & 0 & 0 & 0 & 0 & 0 \\
\hline 24 & 0 & 0 & 0 & 0 & 0 & 0 & 0 & 0 & 0 & 0 & 0 & 0 \\
\hline 25 & 0 & 0 & 0 & 0 & 0 & 0 & 0 & 0 & 0 & 0 & 0 & 0 \\
\hline 26 & 0 & 0 & 0 & 0 & 0 & 0 & 0 & 0 & 0 & 0 & 0 & 0 \\
\hline 27 & 0 & 0 & 0 & 0 & 0 & 0 & 0 & 0 & 0 & 0 & 0 & 0 \\
\hline 28 & 0 & 0 & 0 & 0 & 0 & 0 & 0 & 0 & 0 & 0 & 0 & 0 \\
\hline 29 & 0 & 0 & 0 & 0 & - & 0 & 0 & 0 & 0 & 0 & 0 & 0 \\
\hline 30 & 0 & 0 & 0 & 0 & - & 0 & 0 & 0 & 0 & 0 & 0 & 0 \\
\hline 31 & 0 & 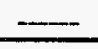 & 0 & 0 & $\cdots$ & 0 & - & 0 & $=$ & 0 & 0 & - \\
\hline Total & 0 & 0 & 0 & 0 & 0 & 0 & 0 & 0 & 0 & 0 & 0 & 0.2 \\
\hline Mean & 0 & 0 & 0 & 0 & 0 & 0 & 0 & 0 & 0 & 0 & 0 & 0.007 \\
\hline Max & 0 & 0 & 0 & 0 & 0 & 0 & 0 & 0 & 0 & 0 & 0 & 0.2 \\
\hline Min & 0 & 0 & 0 & 0 & 0 & 0 & 0 & 0 & 0 & 0 & 0 & 0 \\
\hline AC-FT & 0 & 0 & 0 & 0 & 0 & 0 & 0 & 0 & 0 & 0 & 0 & 0.4 \\
\hline
\end{tabular}

CY 1994: TOTAL* 0.00 MEAN 0 MAX 0 MIN 0 AC-FT 0

WATER YEAR 1995: TOTAL 0.20 MEAN 0.001 MAX 0.2 MIN 0 AC-FT 0.4

* Incomplete Record 


\section{Cañada del Buey at White Rock, NM}

LOCATION. Lat $35^{\circ} 49^{\prime} 39^{\prime \prime}$, long $106^{\circ} 12^{\prime} 40^{\prime \prime}$, in Ramon Vigil Grant, Los Alamos County, 250 mi upstream from NM State Highway 4 in White Rock, NM.

DRAINAGE. AREA.2.14 mi².

PERIOD OF RECORD. October 1991 to September 30, 1995.

GAGE. Data logger with cellular telemetry and concrete control. Elevation of gage is $6,415 \mathrm{ft}$ above National Geodetic Vertical Datum of 1929 from topographic map.

REMARKS. Records fair. No diversions.

EXTREMES FOR PERIOD OF RECORD. Maximum discharge 112 cfs Aug. 14, 1994, gage height $2.40 \mathrm{ft}$. No flow most of the time.

EXTREMES FOR CURRENT WATER YEAR. Maximum discharge $37 \mathrm{cfs}$ at $1900 \mathrm{hrs} \mathrm{Sept.} 7$, gage height $1.97 \mathrm{ft}$. No flow most of the time.

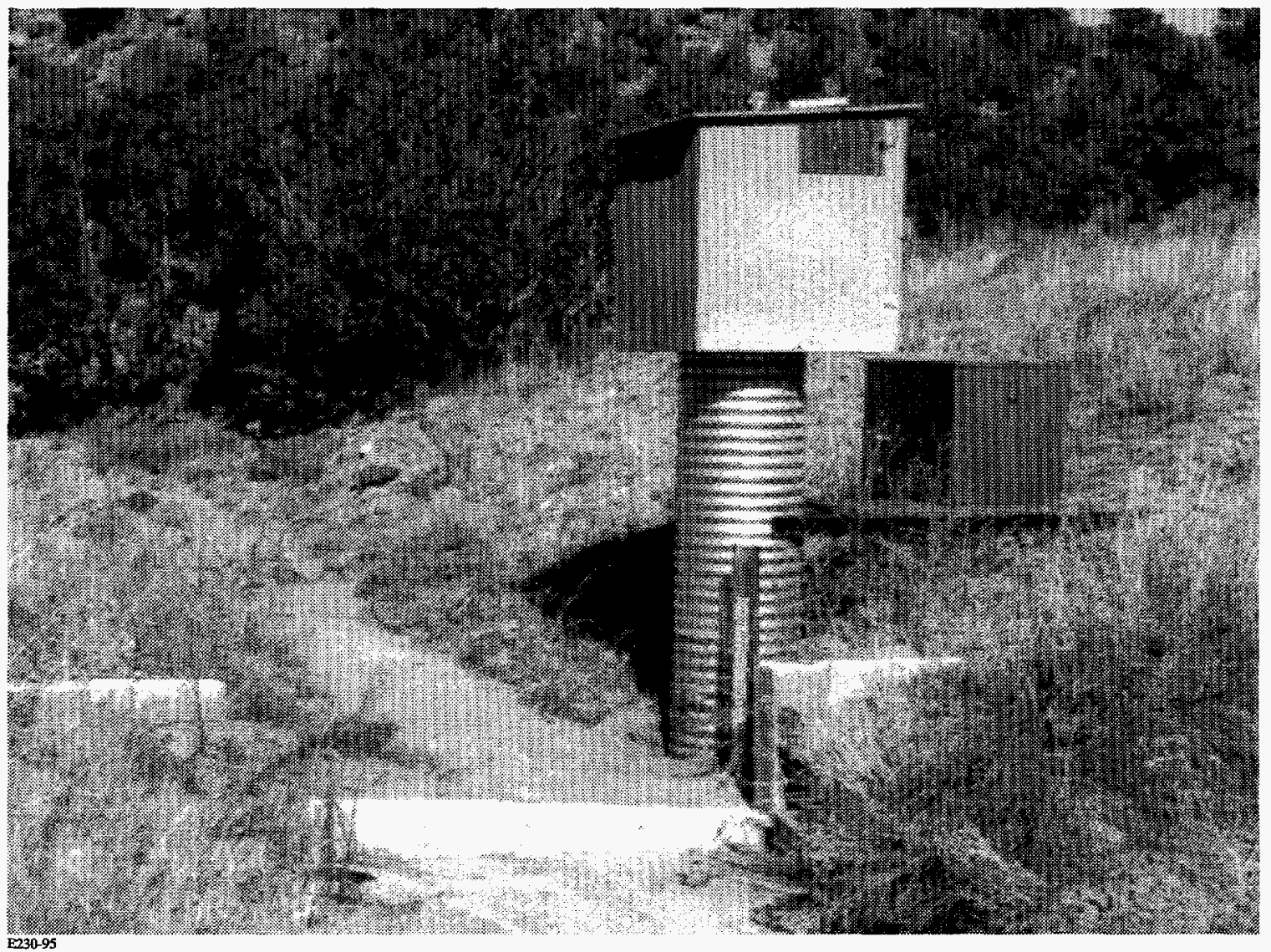


8313230 Cañada del Buey at White Rock, NM

DAILY DISCHARGE IN CUBIC FEET PER SECOND

WATER YEAR OCTOBER 1994 TO SEPTEMBER 1995

\begin{tabular}{|c|c|c|c|c|c|c|c|c|c|c|c|c|}
\hline Day & OCT & NOV & DEC & JAN & FEB & MAR & APR & MAY & JUN & JL & AUG & SEP \\
\hline 1 & 0 & 0 & 0 & 0 & 0 & 0 & 0 & 0 & 0 & 0 & 0 & 0 \\
\hline 2 & 0 & 0 & 0 & 0 & 0 & 0 & 0 & 0 & 0 & 0 & 0 & 0 \\
\hline 3 & 0 & 0 & 0 & 0 & 0 & 0 & 0 & 0 & 0 & 0 & 0 & 0 \\
\hline 4 & 0 & 0 & 0 & 0 & 0 & 0 & 0 & 0 & 0 & 0 & 0 & 0 \\
\hline 5 & 0 & 0 & 0 & 0 & 0 & 0 & 0 & 0 & 0 & 0 & 0 & 0 \\
\hline 6 & 0 & 0 & 0 & 0 & 0 & 0 & 0 & 0 & 0 & 0 & 0 & 0 \\
\hline 7 & 0 & 0 & 0 & 0 & 0 & 0 & 0 & 0 & 0 & 0 & 0 & 1.2 \\
\hline 8 & 0 & 0 & 0 & 0 & 0 & 0 & 0 & 0 & 0 & 0 & 0 & 1.3 \\
\hline 9 & 0 & 0 & 0 & 0 & 0 & 0 & 0 & 0 & 0 & 0 & 0 & 0 \\
\hline 10 & 0 & 0 & 0 & 0 & 0 & 0 & 0 & 0 & 0 & 0 & 0 & 0 \\
\hline 11 & 0 & 0.50 & 0 & 0 & 0 & 0 & 0 & 0 & 0 & 0 & 0.20 & 0 \\
\hline 12 & 0 & 0.50 & 0 & 0 & 0 & 0 & 0 & 0 & 0 & 0 & 0.48 & 0 \\
\hline 13 & 0 & 0 & 0 & 0 & 0.02 & 0 & 0 & 0 & 0 & 0 & 0.72 & 0 \\
\hline 14 & 0 & 0 & 0 & 0 & 0.05 & 0 & 0 & 0 & 0 & 0 & 0.72 & 0.17 \\
\hline 15 & 0 & 0 & 0 & 0 & 0 & 0 & 0 & 0 & 0 & 0 & 0 & 0 \\
\hline 16 & 0 & 0 & 0 & 0 & 0 & 0 & 0 & 0 & 0 & 0 & 0.08 & 0 \\
\hline 17 & 0 & 0 & 0 & 0 & 0 & 0 & 0 & 0 & 0 & 0 & 0.09 & 0 \\
\hline 18 & 0 & 0 & 0 & 0 & 0 & 0 & 0 & 0 & 0 & 0 & 0 & 0 \\
\hline 19 & 0 & 0 & 0 & 0 & 0 & 0 & 0 & 0 & 0 & 0 & 0 & 0 \\
\hline 20 & 0 & 0 & 0 & 0 & 0 & 0 & 0 & 0 & 0 & 0 & 0 & 0 \\
\hline 21 & 0 & 0 & 0 & 0 & 0 & 0 & 0 & 0 & 0 & 0 & 0 & 0 \\
\hline 22 & 0 & 0 & 0 & 0 & 0 & 0 & 0 & 0 & 0 & 0 & 0 & 0 \\
\hline 23 & 0 & 0 & 0 & 0 & 0 & 0 & 0 & 0 & 0 & 0 & 0 & 0 \\
\hline 24 & 0 & 0 & 0 & 0 & 0 & 0 & 0 & 0 & 0 & 0 & 0 & 0 \\
\hline 25 & 0 & 0 & 0 & 0 & 0 & 0 & 0 & 0 & 0 & 0 & 0 & 0 \\
\hline 26 & 0 & 0 & 0 & 0 & 0 & 0 & 0 & 0 & 0 & 0 & 0 & 0 \\
\hline 27 & 0 & 0 & 0 & 0 & 0 & 0 & 0 & 0 & 0 & 0 & 0 & 0 \\
\hline 28 & 0 & 0 & 0 & 0 & 0 & 0 & 0 & 0 & 0 & 0 & 0 & 0 \\
\hline 29 & 0 & 0 & 0 & 0 & - & 0 & 0 & 0.07 & 0 & 0 & 0.85 & 0 \\
\hline 30 & 0 & 0 & 0 & 0 & - & 0 & 0 & 0 & 0 & 0 & 0 & 0 \\
\hline 31 & 0 & $\cdots$ & 0 & 0 & - & 0 & - & 0 & - & 0 & 0 & - \\
\hline Total & 0 & 1 & 0 & 0 & 0.07 & 0 & 0 & 0.07 & 0 & 0 & 3.14 & 2.67 \\
\hline Mean & 0 & 0.033 & 0 & 0 & 0.003 & 0 & 0 & 0.002 & 0 & 0 & 0.1 & 0.089 \\
\hline $\operatorname{Max}$ & 0 & 0.5 & 0 & 0 & 0.05 & 0 & 0 & 0.07 & 0 & 0 & 0.85 & 1.3 \\
\hline Min & 0 & 0 & 0 & 0 & 0 & 0 & 0 & 0 & 0 & 0 & 0 & 0 \\
\hline AC-FT & 0 & 2 & 0 & 0 & 0.1 & 0 & 0 & 0.1 & 0 & 0 & 6.2 & 5.3 \\
\hline
\end{tabular}

CY 1994: TOTAL* 1.00 MEAN 0.011 MAX 0.5 MIN 0 AC-FT 2

WATER YEAR 1995: TOTAL 6.95 MEAN 0.019 MAX 1.3 MIN 0 AC-FT 14

* Incomplete Record 


\section{Pajarito Canyon above Highway 501 near Los Alamos, NM}

LOCATION. Lat $35^{\circ} 52^{\prime} 06^{\prime \prime}$, long $106^{\circ} 21^{\prime} 09^{\prime \prime}$, SE $1 / 4$ NW 1/4, sec. 19, T.19 N, R. 6. E, Los Alamos County, in Santa Fe National Forest, $200 \mathrm{ft}$ upstream from NM State Highway 501.

DRAINAGE AREA. $1.90 \mathrm{mi}^{2}$.

PERIOD OF RECORD. October 1993 to September 30, 1995.

GAGE. Data logger with cellular telemetry and Parshall Flume. Elevation of gage is $7,760 \mathrm{ft}$ above National Geodetic vertical datum of 1929 from topographic map.

REMARKS. Records good.

EXTREMES FOR PERIOD OF RECORD. Maximum discharge $2.4 \mathrm{cfs}$, June 21, 1994, gage height $0.55 \mathrm{ft}$. No flow at times.

EXTREMES FOR CURRENT WATER YEAR. Maximum discharge $1.9 \mathrm{cfs}$ at $1915 \mathrm{hrs,} \mathrm{Nov.}$ 12 gage height $0.74 \mathrm{ft}$. No flow at times.

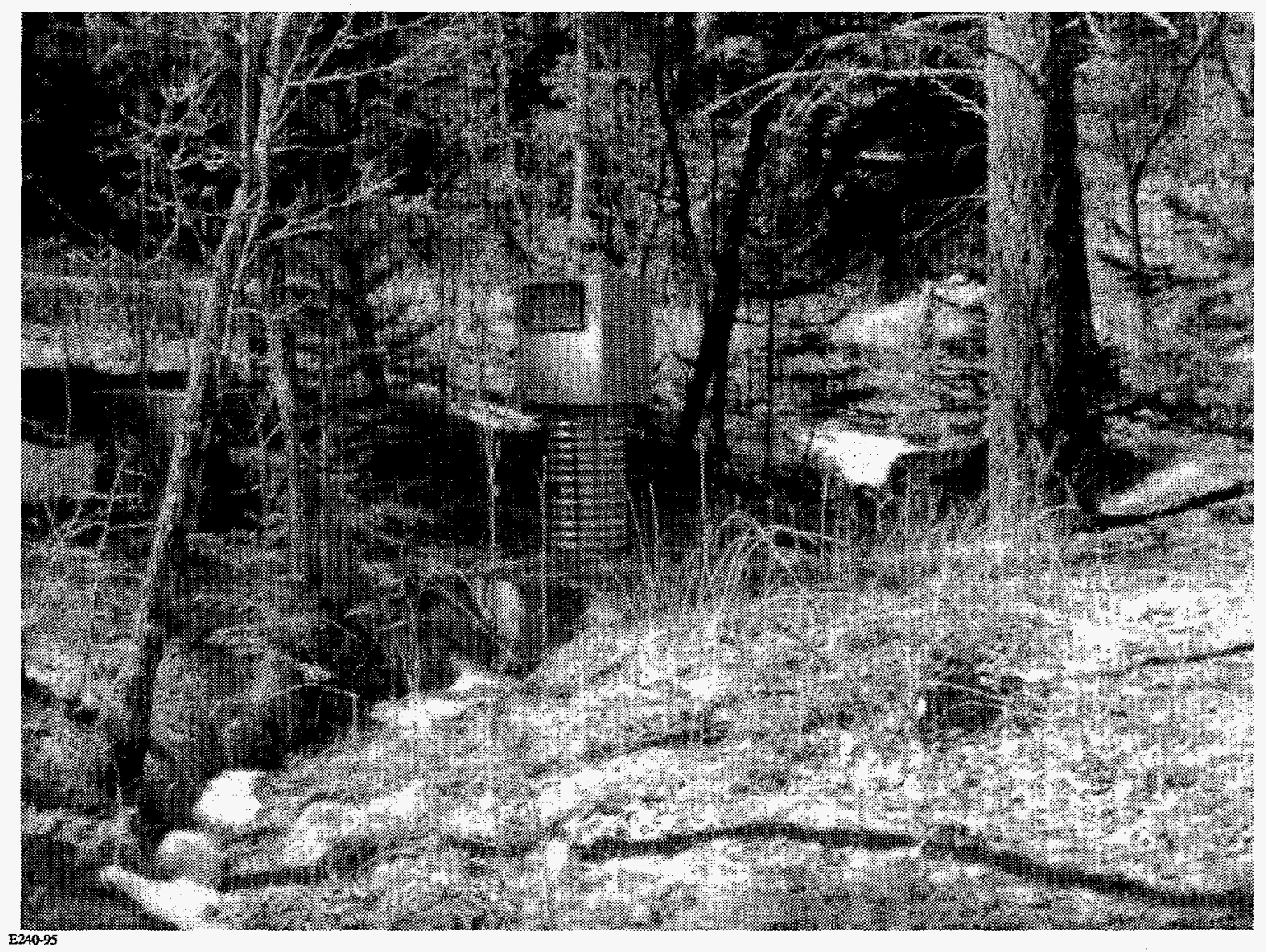


8313240 Pajarito Canyon above Highway 501 near Los Alamos, NM

\begin{tabular}{|c|c|c|c|c|c|c|c|c|c|c|c|c|}
\hline \multirow[b]{2}{*}{ Day } & \multicolumn{12}{|c|}{$\begin{array}{l}\text { DAILY DISCHARGE IN CUBIC FEET PER SECOND } \\
\text { WATER YEAR OCTOBER } 1994 \text { TO SEPTEMBER } 95\end{array}$} \\
\hline & OCT & NOV & DEC & JAN & FEB & MAR & APR & MAY & JUN & JUL & AUG & SEP \\
\hline 1 & 0.12 & 0 & 0 & 0 & 0 & 0 & 0.08 & 0.87 & 0.49 & 0.28 & 0.10 & 0.10 \\
\hline 2 & 0.14 & 0 & 0 & 0 & 0 & 0 & 0.01 & 0.81 & 0.49 & 0.24 & 0.09 & 0.10 \\
\hline 3 & 0.15 & 0 & 0 & 0 & 0 & 0 & 0.01 & 0.84 & 0.49 & 0.23 & 0.09 & 0.09 \\
\hline 4 & 0.17 & 0 & 0 & 0 & 0 & 0 & 0 & 0.83 & 0.47 & 0.23 & 0.09 & 0.09 \\
\hline 5 & 0.15 & 0 & 0 & 0 & 0 & 0.01 & 0 & 0.79 & 0.45 & 0.23 & 0.09 & 0.08 \\
\hline 6 & 0.12 & 0 & 0 & 0 & 0 & 0.18 & 0 & 0.77 & 0.44 & 0.21 & 0.09 & 0.08 \\
\hline 7 & 0.14 & 0 & 0 & 0 & 0 & 0.22 & 0.01 & 0.73 & 0.43 & 0.20 & 0.07 & 0.09 \\
\hline 8 & 0.15 & 0 & 0 & 0 & 0 & 0.05 & 0.05 & 0.68 & 0.42 & 0.18 & 0.06 & 0.10 \\
\hline 9 & 0.12 & 0 & 0 & 0 & 0 & 0.01 & 0.09 & 0.61 & 0.41 & 0.16 & 0.05 & 0.09 \\
\hline 10 & 0.09 & 0 & 0 & 0 & 0 & 0 & 0.11 & 0.57 & 0.39 & 0.15 & 0.04 & 0.09 \\
\hline 11 & 0.09 & 0 & 0 & 0 & 0 & 0 & 0.09 & 0.56 & 0.38 & 0.16 & 0.08 & 0.10 \\
\hline 12 & 0.08 & 0.91 & 0 & 0 & 0 & 0 & 0.07 & 0.59 & 0.35 & 0.15 & 0.07 & 0.09 \\
\hline 13 & 0.09 & 1.3 & 0 & 0 & 0 & 0 & 0.07 & 0.62 & 0.34 & 0.15 & 0.11 & 0.07 \\
\hline 14 & 0.22 & 0.57 & 0 & 0 & 0 & 0 & 0.10 & 0.58 & 0.33 & 0.15 & 0.11 & 0.07 \\
\hline 15 & 0.28 & 0.34 & 0 & 0 & 0 & 0 & 0.14 & 0.51 & 0.32 & 0.15 & 0.08 & 0.07 \\
\hline 16 & 0.24 & 0.22 & 0 & 0 & 0 & 0 & 0.16 & 0.51 & 0.32 & 0.17 & 0.07 & 0.07 \\
\hline 17 & 0.19 & 0.18 & 0 & 0 & 0 & 0.02 & 0.19 & 0.51 & 0.49 & 0.17 & 0.06 & 0.05 \\
\hline 18 & 0.08 & 0.29 & 0 & 0 & 0 & 0.08 & 0.18 & 0.52 & 0.38 & 0.25 & 0.06 & 0.04 \\
\hline 19 & 0.04 & 0.16 & 0 & 0 & 0 & 0.09 & 0.17 & 0.48 & 0.35 & 0.19 & 0.09 & 0.04 \\
\hline 20 & 0.02 & 0.20 & 0 & 0 & 0 & 0.11 & 0.16 & 0.45 & 0.33 & 0.16 & 0.08 & 0.04 \\
\hline 21 & 0.02 & 0.06 & 0 & 0 & 0 & 0.11 & 0.17 & 0.44 & 0.32 & 0.14 & 0.06 & 0.03 \\
\hline 22 & 0.01 & 0.04 & 0 & 0 & 0 & 0.13 & 0.10 & 0.41 & 0.31 & 0.13 & 0.08 & 0.03 \\
\hline 23 & 0.01 & 0.02 & 0 & 0 & 0 & 0.09 & 0.19 & 0.41 & 0.29 & 0.13 & 0.08 & 0.03 \\
\hline 24 & 0.01 & 0.02 & 0 & 0 & 0 & 0.06 & 0.18 & 0.44 & 0.27 & 0.12 & 0.12 & 0.03 \\
\hline 25 & 0.01 & 0.01 & 0 & 0 & 0 & 0.06 & 0.20 & 0.44 & 0.29 & 0.12 & 0.27 & 0.03 \\
\hline 26 & 0.01 & 0.01 & 0 & 0 & 0 & 0.04 & 0.31 & 0.44 & 0.27 & 0.11 & 0.25 & 0.03 \\
\hline 27 & 0 & 0 & 0 & 0 & 0 & 0.01 & 0.59 & 0.47 & 0.26 & 0.11 & 0.24 & 0.03 \\
\hline 28 & 0 & 0 & 0 & 0 & 0 & 0.01 & 0.75 & 0.51 & 0.27 & 0.10 & 0.18 & 0.04 \\
\hline 29 & 0 & 0 & 0 & 0 & $\ldots$ & 0 & 0.86 & 0.59 & 0.26 & 0.10 & 0.19 & 0.06 \\
\hline 30 & 0 & 0 & 0 & 0 & - & 0 & 0.89 & 0.54 & 0.28 & 0.10 & 0.13 & 0.04 \\
\hline 31 & 0 & - & 0 & 0 & - & 0.03 & - & 0.52 & - & 0.10 & 0.11 & - \\
\hline Total & 2.75 & 4.33 & 0 & 0 & 0 & 1.31 & 5.93 & 18.04 & 10.89 & 5.07 & 3.29 & 1.9 \\
\hline Mean & 0.089 & 0.14 & 0 & 0 & 0 & 0.042 & 0.2 & 0.58 & 0.36 & 0.16 & 0.11 & 0.063 \\
\hline $\operatorname{Max}$ & 0.28 & 1.3 & 0 & 0 & 0 & 0.22 & 0.89 & 0.87 & 0.49 & 0.28 & 0.27 & 0.1 \\
\hline Min & 0 & 0 & 0 & 0 & 0 & 0 & 0 & 0.41 & 0.26 & 0.1 & 0.04 & 0.03 \\
\hline $\begin{array}{l}\text { AC- } \\
\text { FT }\end{array}$ & 5.5 & 8.6 & 0 & 0 & 0 & 2.6 & 12 & 36 & 22 & 10 & 6.5 & 3.8 \\
\hline
\end{tabular}

CY1994: TOTAL* 7.08 MEAN 0.077 MAX 1.3 MIN 0 AC-FT 14

WATER YEAR 1995: TOTAL 53.51 MEAN 0.15 MAX 1.3 MIN 0 AC-FT 106

* Incomplete Record 


\section{Pajarito Canyon above TA-18 near Los Alamos, NM}

LOCATION. Lat $35^{\circ} 50^{\prime} 59^{\prime \prime}$, long $106^{\circ} 17^{\prime} 02^{\prime \prime}$, Ramon Vigil Grant, Los Alamos County, $1.5 \mathrm{mi}$ upstream from LANL TA-18 and Three-Mile Canyon and $.15 \mathrm{mi}$ SE of Pajarito Road.

DRAINAGE AREA. $7.84 \mathrm{mi}^{2}$.

PERIOD OF RECORD. November 1993 to September 30, 1995.

GAGE. Data logger with cellular telemetry and concrete control. Elevation of gage is $6,865 \mathrm{ft}$. above National Geodetic Vertical Datum of 1929 from topographic map.

REMARKS. Record fair.

EXTREMES FOR PERIOD OF RECORD. Maximum discharge, $26 \mathrm{cfs}$, June 21, 1994, gage height $2.40 \mathrm{ft}$. No flow at times.

EXTREMES FOR CURRENT WATER YEAR. Maximum discharge, 24 cfs, 1500 hrs, Aug. 29, gage height $2.24 \mathrm{ft}$. No flow at times.

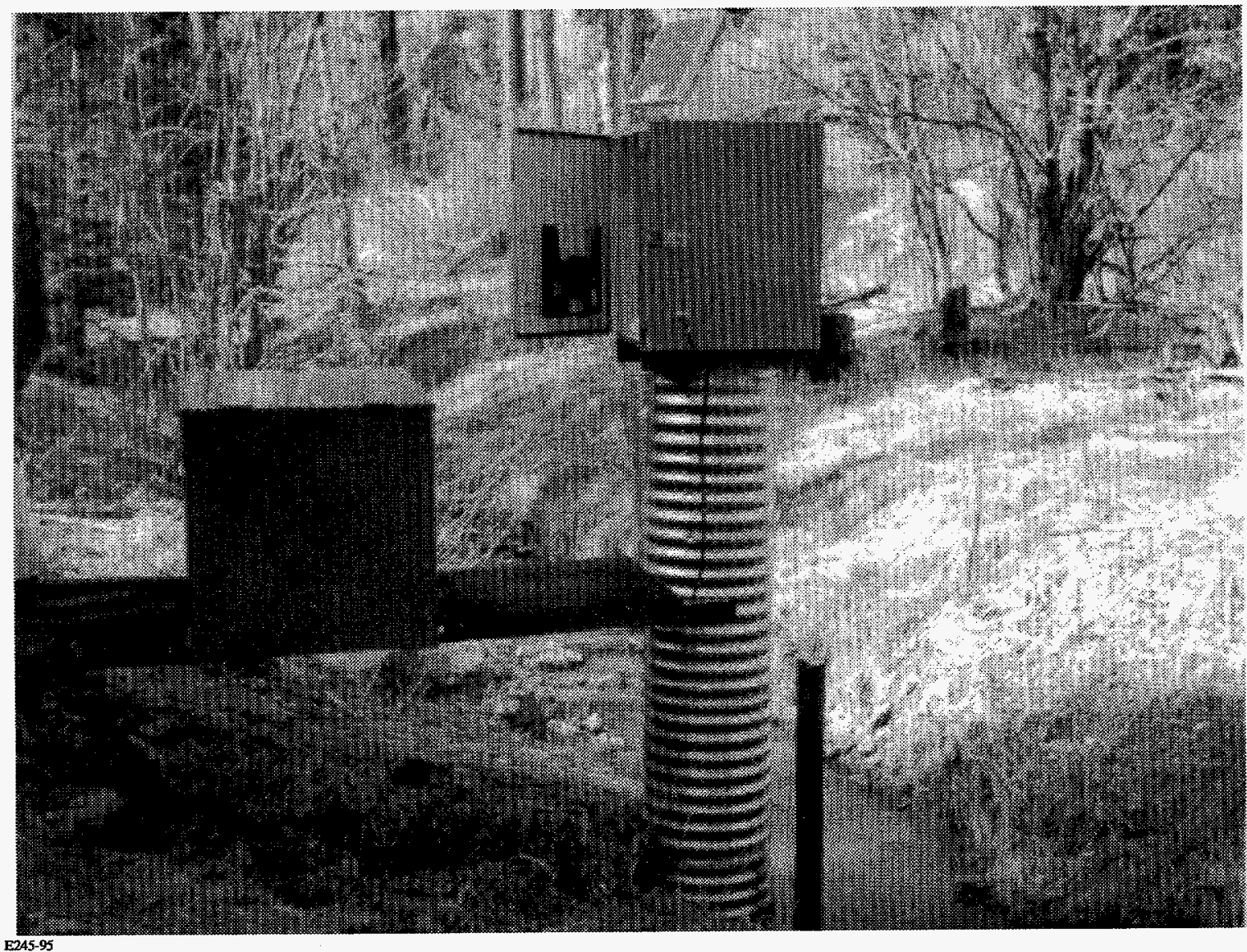




\section{Pajarito Canyon above TA-18 near Los Alamos, NM}

DAILY DISCHARGE IN CUBIC FEET PER SECOND

WATER YEAR OCTOBER 1994 TO SEPTEMBER 1995

\begin{tabular}{|c|c|c|c|c|c|c|c|c|c|c|c|c|}
\hline Day & OCT & NOV & DEC & JAN & FEB & MAR & APR & MAY & JUN & JUL & AUG & SEP \\
\hline 1 & 0.22 & 0.47 & 0 & 0 & 0 & 0.82 & 0.65 & 0.93 & 2.1 & 0.50 & 0 & 0.08 \\
\hline 2 & 0.22 & 0.47 & 0 & 0 & 0 & 1.1 & 0.58 & 0.92 & 1.9 & 0.40 & 0 & 0.03 \\
\hline 3 & 0.22 & 0.47 & 0 & 0 & 0 & 0.96 & 0.56 & 0.89 & 1.7 & 0.35 & 0 & 0 \\
\hline 4 & 0.22 & 0.48 & 0 & 0 & 0 & 1.4 & 0.52 & 0.89 & 1.6 & 0.33 & 0.02 & 0 \\
\hline 5 & 0.22 & 0.50 & 0 & 0 & 0 & 1.5 & 0.50 & 0.95 & 1.5 & 0.30 & 0.33 & 0 \\
\hline 6 & 0.23 & 0.55 & 0 & 0 & 0 & 2.7 & 0.48 & 0.89 & 1.5 & 0.21 & 0 & 0.01 \\
\hline 7 & 0.24 & 0.60 & 0 & 0 & 0 & 1.6 & 0.43 & 0.87 & 1.4 & 0.18 & 0 & 0.83 \\
\hline 8 & 0.25 & 0.70 & 0 & 0 & 0 & 1.1 & 0.42 & 0.86 & 1.3 & 0.16 & 0 & 0.44 \\
\hline 9 & 0.25 & 0.80 & 0 & 0 & 0 & 1.0 & 0.44 & 0.81 & 1.2 & 0.13 & 0 & 0.35 \\
\hline 10 & 0.25 & 1.0 & 0 & 0 & 0 & 1.0 & 0.50 & 0.77 & 1.2 & 0.10 & 0 & 0.14 \\
\hline 11 & 0.29 & 0.90 & 0 & 0 & 0 & 0.98 & 0.72 & 0.75 & 1.1 & 0.09 & 0.08 & 0.36 \\
\hline 12 & 0.32 & 0.70 & 0 & 0 & 0 & 0.95 & 0.58 & 0.72 & 1.1 & 0.08 & 0 & 0.07 \\
\hline 13 & 0.40 & 0.60 & 0 & 0 & 0.01 & 0.90 & 0.57 & 0.72 & 1.1 & 0.08 & 0.14 & 0.03 \\
\hline 14 & 0.50 & 0.40 & 0 & 0 & 0.02 & 0.90 & 0.55 & 0.70 & 1.0 & 0.08 & 0.09 & 0 \\
\hline 15 & 0.70 & 0.20 & 0 & 0 & 0.02 & 0.88 & 0.53 & 0.69 & 0.97 & 0.11 & 0.04 & 0 \\
\hline 16 & 0.80 & 0.11 & 0 & 0 & 0.03 & 0.85 & 0.51 & 0.67 & 0.95 & 0.35 & 0 & 0 \\
\hline 17 & 1.00 & 0.06 & 0 & 0 & 0.05 & 0.85 & 0.53 & 0.67 & 0.95 & 0.30 & 0 & 0 \\
\hline 18 & 0.90 & 0 & 0 & 0 & 0.06 & 0.84 & 0.54 & 0.74 & 0.69 & 1.2 & 0 & 0 \\
\hline 19 & 0.80 & 0 & 0 & 0 & 0.07 & 0.84 & 0.57 & 0.67 & 0.56 & 0.46 & 0 & 0 \\
\hline 20 & 0.80 & 0 & 0 & 0 & 0.08 & 0.84 & 0.58 & 0.64 & 0.51 & 0.35 & 0.03 & 0 \\
\hline 21 & 0.70 & 0 & 0 & 0 & 0.09 & 0.84 & 0.60 & 0.64 & 0.49 & 0.27 & 0 & 0 \\
\hline 22 & 0.65 & 0 & 0 & 0 & 0.10 & 0.82 & 0.65 & 0.64 & 0.43 & 0.22 & 0 & 0 \\
\hline 23 & 0.60 & 0 & 0 & 0 & 0.20 & 0.84 & 0.65 & 0.65 & 0.41 & 0.14 & 0 & 0 \\
\hline 24 & 0.55 & 0 & 0 & 0 & 0.30 & 0.84 & 0.71 & 0.66 & 0.41 & 0.08 & 0 & 0 \\
\hline 25 & 0.55 & 0 & 0 & 0 & 0.42 & 0.84 & 0.75 & 0.59 & 0.44 & 0.04 & 0.02 & 0 \\
\hline 26 & 0.55 & 0 & 0 & 0 & 0.50 & 0.82 & 0.75 & 0.59 & 0.46 & 0.03 & 0 & 0 \\
\hline 27 & 0.50 & 0 & 0 & 0 & 0.55 & 0.92 & 0.75 & 0.57 & 0.45 & 0.02 & 0.12 & 0 \\
\hline 28 & 0.50 & 0 & 0 & 0 & 0.79 & 0.75 & 0.80 & 0.61 & 0.61 & 0.01 & 0.08 & 0 \\
\hline 29 & 0.47 & 0 & 0 & 0 & - & 0.72 & 0.89 & 3.4 & 0.55 & 0.01 & 1.3 & 0 \\
\hline 30 & 0.47 & 0 & 0 & 0 & - & 0.66 & 0.91 & 3.8 & 0.54 & 0.01 & 0.35 & 0 \\
\hline 31 & 0.47 & - & 0 & 0 & - & 0.73 & - & 2.9 & - & 0 & 0.17 & - \\
\hline Total & 14.84 & 9.01 & 0 & 0 & 3.29 & 30.79 & 18.22 & 30.8 & $\begin{array}{l}29.1 \\
2\end{array}$ & 6.59 & 2.77 & 2.34 \\
\hline Mean & 0.48 & 0.3 & 0 & 0 & 0.12 & 0.99 & 0.61 & 0.99 & 0.97 & 0.21 & 0.089 & 0.078 \\
\hline $\operatorname{Max}$ & 1 & 1 & 0 & 0 & 0.79 & 2.7 & 0.91 & 3.8 & 2.1 & 1.2 & 1.3 & 0.83 \\
\hline Min & 0.22 & 0 & 0 & 0 & 0 & 0.66 & 0.42 & 0.57 & 0.41 & 0 & 0 & 0 \\
\hline AC-FT & 29 & 18 & 0 & 0 & 6.5 & 61 & 36 & 61 & 58 & 13 & 5.5 & 4.6 \\
\hline
\end{tabular}

CY 1994: TOTAL* 23.85 MEAN 0.26 MAX 1.0 MIN 0 AC-FT 47

WATER YEAR 1995: TOTAL 147.7 MEAN 0.4 MAX 3.8 MIN 0 AC-FT 293

* Incomplete Record 


\section{Pajarito Canyon above Highway 4 near White Rock, NM}

LOCATION. Lat $35^{\circ} 49^{\prime} 28^{\prime \prime}$, long $106^{\circ} 13^{\prime} 36^{\prime \prime}$, in Ramon Vigil Grant, Los Alamos County, 0.25 mi upstream from NM State Highway 4 and White Rock, NM

DRAINAGE AREA. $10.9 \mathrm{mi}^{2}$.

PERIOD OF RECORD. November 1993 to September 30, 1995.

GAGE. Data logger with cellular telemetry and concrete control. Elevation of gage is $6,550 \mathrm{ft}$ above National Geodetic Vertical Datum of 1929 from topographic map and GPS.

REMARKS. Records poor.

EXTREMES FOR PERIOD OF RECORD. Maximum discharge $4.6 \mathrm{cfs}$ Aug. 29, 1995, gage height $2.76 \mathrm{ft}$. No flow at times.

EXTREMES FOR CURRENT WATER YEAR. Maximum discharge $4.6 \mathrm{cfs}$ at $1430 \mathrm{hrs}$ Aug. 29 , gage height $2.76 \mathrm{ft}$. No flow at times.

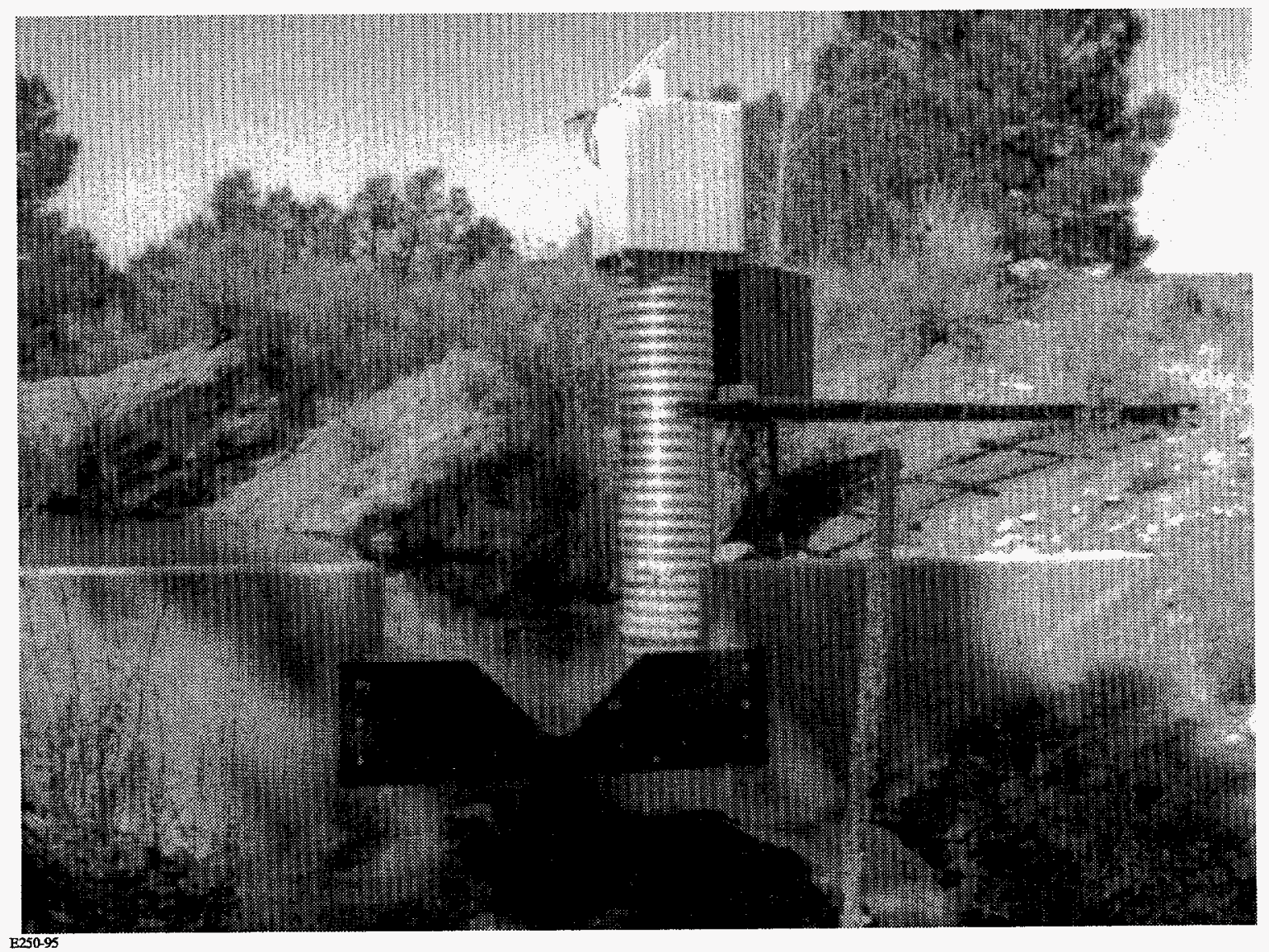


8313250 Pajarito Canyon above Highway 4 near White Rock, NM

\begin{tabular}{|c|c|c|c|c|c|c|c|c|c|c|c|c|}
\hline \multirow[b]{2}{*}{ Day. } & \multicolumn{12}{|c|}{$\begin{array}{l}\text { DAILY DISCHARGE IN CUBIC FEET PER SECOND } \\
\text { WATER YEAR OCTOBER } 1994 \text { TO SEPTEMBER } 1995\end{array}$} \\
\hline & OCT & NOV & DEC & JAN & FEB & MAR & APR & MAY & $\pi \mathrm{N}$ & JUL & AUG & SEP \\
\hline 1 & 0.10 & 0 & 0.09 & 0 & 0 & 0.05 & 0.06 & 0.05 & 0.12 & 0.05 & 0 & 0 \\
\hline 2 & 0.10 & 0 & 0.09 & 0 & 0 & 0.06 & 0.06 & 0.05 & 0.11 & 0.02 & 0 & 0 \\
\hline 3 & 0.10 & 0 & 0.09 & 0 & 0 & 0.06 & 0.06 & 0.05 & 0.09 & 0.01 & 0 & 0 \\
\hline 4 & 0.11 & 0 & 0.09 & 0 & 0 & 0.06 & 0.06 & 0.05 & 0.09 & 0 & 0 & 0 \\
\hline 5 & 0.11 & 0 & 0.09 & 0 & 0 & 0.06 & 0.06 & 0.06 & 0.08 & 0 & 0 & 0 \\
\hline 6 & 0.11 & 0 & 0.09 & 0 & 0.01 & 0.06 & 0.06 & 0.06 & 0.07 & 0 & 0 & 0 \\
\hline 7 & 0.12 & 0 & 0.09 & 0 & 0.10 & 0.06 & 0.06 & 0.05 & 0.06 & 0 & 0 & 0.03 \\
\hline 8 & 0.12 & 0.10 & 0.09 & 0 & 0.10 & 0.06 & 0.06 & 0.07 & 0.06 & 0 & 0 & 0.10 \\
\hline 9 & 0.12 & 0.70 & 0.09 & 0 & 0.10 & 0.06 & 0.06 & 0.06 & 0.06 & 0 & 0 & 0.06 \\
\hline 10 & 0.12 & 0.65 & 0.09 & 0 & 0.09 & 0.06 & 0.06 & 0.05 & 0.05 & 0 & 0 & 0.02 \\
\hline 11 & 0.12 & 0.45 & 0.09 & 0 & 0.08 & 0.06 & 0.06 & 0.06 & 0.05 & 0 & 0 & 0.02 \\
\hline 12 & 0.12 & 0.40 & 0.09 & 0 & 0.08 & 0.06 & 0.06 & 0.05 & 0.05 & 0 & 0.01 & 0.01 \\
\hline 13 & 0.12 & 0.40 & 0.09 & 0 & 0.12 & 0.06 & 0.06 & 0.05 & 0.05 & 0 & 0.13 & 0.01 \\
\hline 14 & 0.20 & 0.40 & 0.09 & 0 & 0.15 & 0.06 & 0.06 & 0.05 & 0.05 & 0 & 0.05 & 0.01 \\
\hline 15 & 0.60 & 0.30 & 0.10 & 0 & 0.14 & 0.06 & 0.06 & 0.05 & 0.04 & 0 & 0.02 & 0.02 \\
\hline 16 & 1.1 & 0.25 & 0.10 & 0 & 0.10 & 0.06 & 0.06 & 0.04 & 0.03 & 0 & 0.01 & 0.01 \\
\hline 17 & 2.0 & 0.20 & 0.10 & 0 & 0.08 & 0.06 & 0.06 & 0.05 & 0.02 & 0 & 0.02 & 0.01 \\
\hline 18 & 0.40 & 0.20 & 0.10 & 0 & 0.08 & 0.06 & 0.06 & 0.05 & 0.02 & 0.10 & 0.02 & 0.01 \\
\hline 19 & 0.20 & 0.20 & 0.10 & 0 & 0.06 & 0.06 & 0.06 & 0.05 & 0.01 & 0.03 & 0.01 & 0.01 \\
\hline 20 & 0.13 & 0.12 & 0.10 & 0 & 0.06 & 0.06 & 0.06 & 0.05 & 0.01 & 0.03 & 0.01 & 0 \\
\hline 21 & 0.10 & 0.10 & 0.10 & 0 & 0.05 & 0.06 & 0.06 & 0.04 & 0 & 0.03 & 0 & 0 \\
\hline 22 & 0.09 & 0.09 & 0.10 & 0 & 0.06 & 0.06 & 0.06 & 0.04 & 0 & 0.03 & 0 & 0 \\
\hline 23 & 0.08 & 0.08 & 0.09 & 0 & 0.05 & 0.06 & 0.06 & 0.04 & 0 & 0 & 0 & 0 \\
\hline 24 & 0.07 & 0.08 & 0.09 & 0 & 0.03 & 0.06 & 0.06 & 0.09 & 0.01 & 0 & 0 & 0 \\
\hline 25 & 0.04 & 0.09 & 0.09 & 0 & 0.04 & 0.06 & 0.06 & 0.07 & 0.02 & 0 & 0 & 0 \\
\hline 26 & 0.02 & 0.09 & 0.09 & 0 & 0.05 & 0.06 & 0.06 & 0.07 & 0.06 & 0 & 0 & 0 \\
\hline 27 & 0.02 & 0.09 & 0.09 & 0 & 0.04 & 0.06 & 0.05 & 0.06 & 0.04 & 0 & 0 & 0 \\
\hline 28 & 0 & 0.09 & 0.09 & 0 & 0.03 & 0.06 & 0.05 & 0.09 & 0.03 & 0 & 0 & 0 \\
\hline 29 & 0 & 0.09 & 0.09 & 0 & - & 0.06 & 0.05 & 0.31 & 0.06 & 0 & 0.3 & 0.01 \\
\hline 30 & 0 & 0.09 & 0.09 & 0 & $\ldots$ & 0.06 & 0.05 & 0.48 & 0.09 & 0 & 0.03 & 0.01 \\
\hline 31 & 0 & $=$ & 0.09 & 0 & - & 0.06 & - & 0.14 & - & 0 & 0.01 & - \\
\hline Total & 6.52 & 5.26 & 2.87 & 0 & 1.7 & 1.85 & 1.76 & 2.48 & 1.43 & 0.3 & 0.62 & 0.34 \\
\hline Mean & 0.21 & 0.18 & 0.093 & 0 & 0.061 & 0.06 & 0.059 & 0.08 & 0.048 & 0.01 & 0.02 & 0.011 \\
\hline $\operatorname{Max}$ & 2.0 & 0.70 & 0.10 & 0 & 0.15 & 0.06 & 0.06 & 0.48 & 0.12 & 0.10 & 0.30 & 0.10 \\
\hline Min & 0 & 0 & 0.09 & 0 & 0 & 0.05 & 0.05 & 0.04 & 0 & 0 & 0 & 0 \\
\hline AC-FT & 13 & 10 & 5.7 & 0 & 3.4 & 3.7 & 3.5 & 4.9 & 2.8 & 0.6 & 1.2 & 0.7 \\
\hline
\end{tabular}

CY 1994: TOTAL*14.65 MEAN 0.16 MAX 2.0 MIN 0 AC-FT 29

WATER YEAR 1995: TOTAL 25.13 MEAN 0.069 MAX 2.0 MIN 0 AC-FT 50

* Incomplete Record 


\section{Water Canyon above Highway 501 near Los Alamos, NM}

LOCATION. Lat $35^{\circ} 50^{\prime} 11^{\prime \prime}$, long $106^{\circ} 21^{\prime} 46^{\prime \prime}$, T. 19 N, R. 5 E., Los Alamos County in Santa Fe National Forest, $0.3 \mathrm{mi}$ upstream from NM State Highway 501 and $0.3 \mathrm{mi}$ NW of junction of State Highways 501 and 4.

DRAINAGE AREA. $3.39 \mathrm{mi}^{2}$.

PERIOD OF RECORD. October 1994 to September 30, 1995.

GAGE. Data logger with cellular telemetry and 120-degree weir plate. Elevation of gage is 7,575 $\mathrm{ft}$ above National Geodetic Vertical Datum of 1929 from topographic map.

REMARKS. Records good.

EXTREMES FOR CURRENT WATER YEAR. Maximum discharge $0.21 \mathrm{cfs}$ at $1335 \mathrm{hrs,} \mathrm{March}$ 14 gage height $1.30 \mathrm{ft}$. No flow most of time.

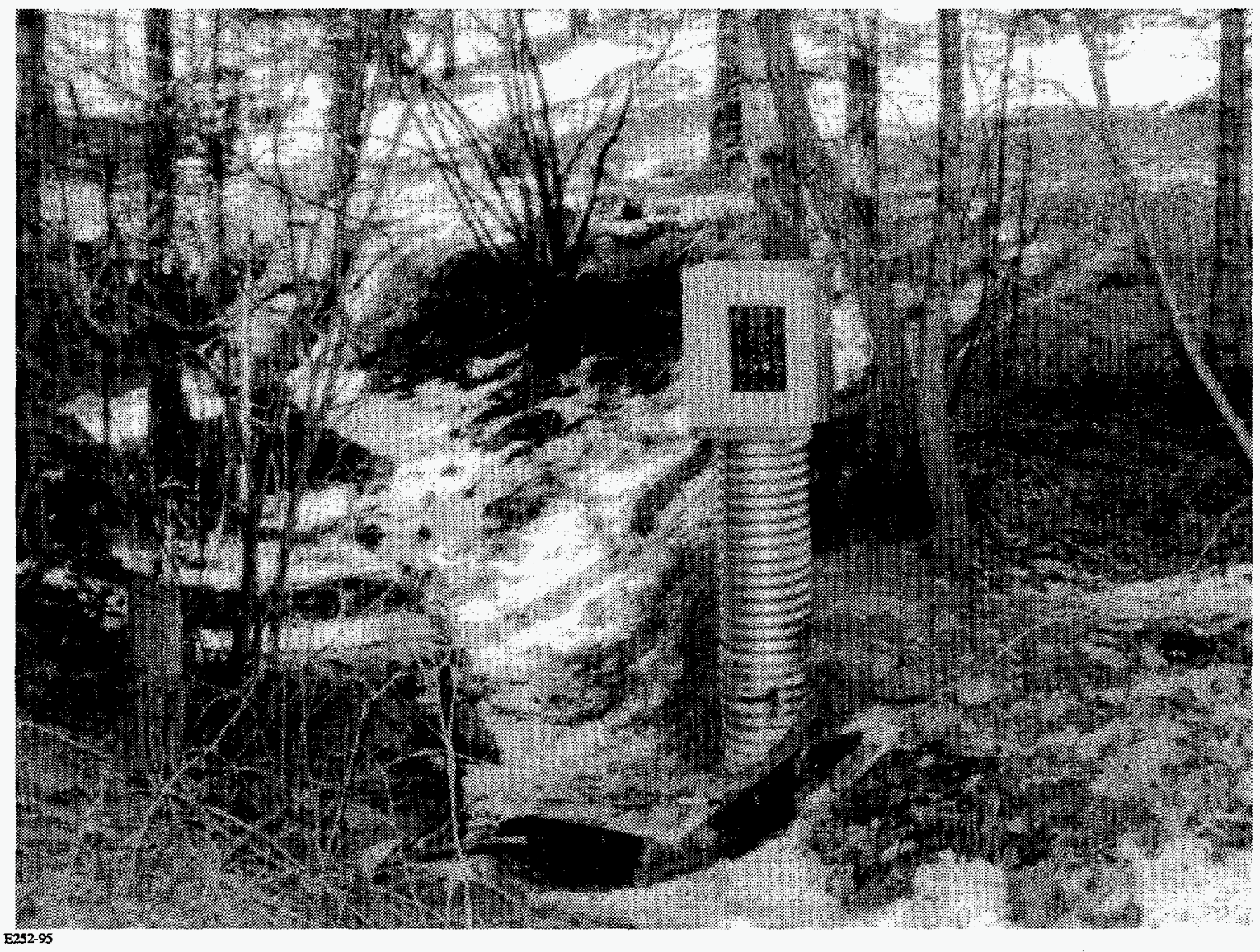


DISCHARGE IN CUBIC FEET PER SECOND

WATER YEAR OCTOBER 1994 TO SEPTEMBER 1995

\begin{tabular}{|c|c|c|c|c|c|c|c|c|c|c|c|c|}
\hline Day & OCT & NOV & DEC & JAN & FEB & MAR & APR & MAY & JUN & JUL & AUG & SEP \\
\hline 1 & 0 & 0 & 0 & 0 & 0 & 0 & 0.10 & 0 & 0 & 0 & 0 & 0 \\
\hline 2 & 0 & 0 & 0 & 0 & 0 & 0 & 0.09 & 0 & 0 & 0 & 0 & 0 \\
\hline 3 & 0 & 0 & 0 & 0 & 0 & 0 & 0.09 & 0 & 0 & 0 & 0 & 0 \\
\hline 4 & 0 & 0 & 0 & 0 & 0 & 0 & 0.08 & 0 & 0 & 0 & 0 & 0 \\
\hline 5 & 0 & 0 & 0 & 0 & 0 & 0 & 0.08 & 0 & 0 & 0 & 0 & 0 \\
\hline 6 & 0 & 0 & 0 & 0 & 0 & 0 & 0.07 & 0.01 & 0 & 0 & 0 & 0 \\
\hline 7 & 0 & 0 & 0 & 0 & 0 & 0 & 0.06 & 0.02 & 0 & 0 & 0 & 0 \\
\hline 8 & 0 & 0 & 0 & 0 & 0 & 0 & 0.05 & 0.02 & 0 & 0 & 0 & 0 \\
\hline 9 & 0 & 0 & 0 & 0 & 0 & 0 & 0.04 & 0.04 & 0 & 0 & 0 & 0 \\
\hline 10 & 0 & 0 & 0 & 0 & 0 & 0.05 & 0.04 & 0.05 & 0 & 0 & 0 & 0 \\
\hline 11 & 0 & 0 & 0 & 0 & 0 & 0.06 & 0.04 & 0.06 & 0 & 0 & 0 & 0 \\
\hline 12 & 0 & 0 & 0 & 0 & 0 & 0.06 & 0.04 & 0.07 & 0 & 0 & 0 & 0 \\
\hline 13 & 0 & 0 & 0 & 0 & 0 & 0.05 & 0.03 & 0.08 & 0 & 0 & 0 & 0 \\
\hline 14 & 0 & 0 & 0 & 0 & 0 & 0.08 & 0.03 & 0.07 & 0 & 0 & 0 & 0 \\
\hline 15 & 0 & 0 & 0 & 0 & 0 & 0.12 & 0.02 & 0.05 & 0 & 0 & 0 & 0 \\
\hline 16 & 0 & 0 & 0 & 0 & 0 & 0.14 & 0.02 & 0.05 & 0 & 0 & 0 & 0 \\
\hline 17 & 0 & 0 & 0 & 0 & 0.04 & 0.15 & 0.01 & 0.05 & 0 & 0 & 0 & 0 \\
\hline 18 & 0 & 0 & 0 & 0 & 0.12 & 0.15 & 0.01 & 0.05 & 0 & 0 & 0 & 0 \\
\hline 19 & 0 & 0 & 0 & 0 & 0.12 & 0.15 & 0.01 & 0.05 & 0 & 0 & 0 & 0 \\
\hline 20 & 0 & 0 & 0 & 0 & 0.10 & 0.14 & 0.01 & 0.04 & 0 & 0 & 0 & 0 \\
\hline 21 & 0 & 0 & 0 & 0 & 0.02 & 0.14 & 0.01 & 0.04 & 0 & 0 & 0 & 0 \\
\hline 22 & 0 & 0 & 0 & 0 & 0 & 0.14 & 0 & 0.04 & 0 & 0 & 0 & 0 \\
\hline 23 & 0 & 0 & 0 & 0 & 0 & 0.13 & 0 & 0.03 & 0 & 0 & 0 & 0 \\
\hline 24 & 0 & 0 & 0 & 0 & 0 & 0.13 & 0 & 0.03 & 0 & 0 & 0 & 0 \\
\hline 25 & 0 & 0 & 0 & 0 & 0 & 0.13 & 0 & 0.03 & 0 & 0 & 0 & 0 \\
\hline 26 & 0 & 0 & 0 & 0 & 0 & 0.13 & 0 & 0.02 & 0 & 0 & 0 & 0 \\
\hline 27 & 0 & 0 & 0 & 0 & 0 & 0.12 & 0 & 0.02 & 0 & 0 & 0 & 0 \\
\hline 28 & 0 & 0 & 0 & 0 & 0 & 0.12 & 0 & 0.02 & 0 & 0 & 0 & 0 \\
\hline 29 & 0 & 0 & 0 & 0 & $\ldots$ & 0.11 & 0 & 0.01 & 0 & 0 & 0 & 0 \\
\hline 30 & 0 & 0 & 0 & 0 & - & 0.11 & 0 & 0.01 & 0 & 0 & 0 & 0 \\
\hline 31 & 0 & - & 0 & 0 & - & 0.10 & - & 0.01 & $=$ & 0 & 0 & 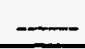 \\
\hline Total & 0 & 0 & 0 & 0 & 0.4 & 2.51 & 0.93 & 0.97 & 0 & 0 & 0 & 0 \\
\hline Mean & 0 & 0 & 0 & 0 & 0.014 & 0.081 & 0.03 & 0.031 & 0 & 0 & 0 & 0 \\
\hline $\operatorname{Max}$ & 0 & 0 & 0 & 0 & 0.12 & 0.15 & 0.10 & 0.08 & 0 & 0 & 0 & 0 \\
\hline Min & 0 & 0 & 0 & 0 & 0 & 0 & 0 & 0 & 0 & 0 & 0 & 0 \\
\hline AC-FT & 0 & 0 & 0 & 0 & 0.8 & 5 & 1.8 & 1.9 & 0 & 0 & 0 & 0 \\
\hline
\end{tabular}

CY 1994: TOTAL* 0.00 MEAN 0 MAX 0 MIN 0 AC-FT 0

WATER YEAR 1995: TOTAL 4.81 MEAN 0.013 MAX 0.15 MIN 0 AC-FT 9.5

* Incomplete Record 


\section{Cañon del Valle above Highway 501 near Los Alamos, NM}

LOCATION. Lat $35^{\circ} 51^{\prime} 06^{\prime \prime}$, long $106^{\circ} 21^{\prime} 17^{\prime \prime}$, NE 1/4, NE 1/4, sec. 25 , T.19 N, R. 5 E., Los Alamos County in Santa Fe National Forest, 0.25 mi upstream from NM State Highway 501, 4.7 mi above mouth and $1.5 \mathrm{mi}$ of junction of State Highways 501 and 4.

DRAINAGE AREA. $2.46 \mathrm{mi}^{2}$.

PERIOD OF RECORD. October 1994 to September 30, 1995.

GAGE. Data logger with cellular telemetry and $120^{\circ}$ weir plate. Elevation of gage is $7,745 \mathrm{ft}$ above National Geodetic Vertical Datum of 1929 from topographic map.

REMARKS. Records fair.

EXTREMES FOR PERIOD OF RECORD. No flow for period.

EXTREMES FOR CURRENT WATER YEAR. No flow all year.

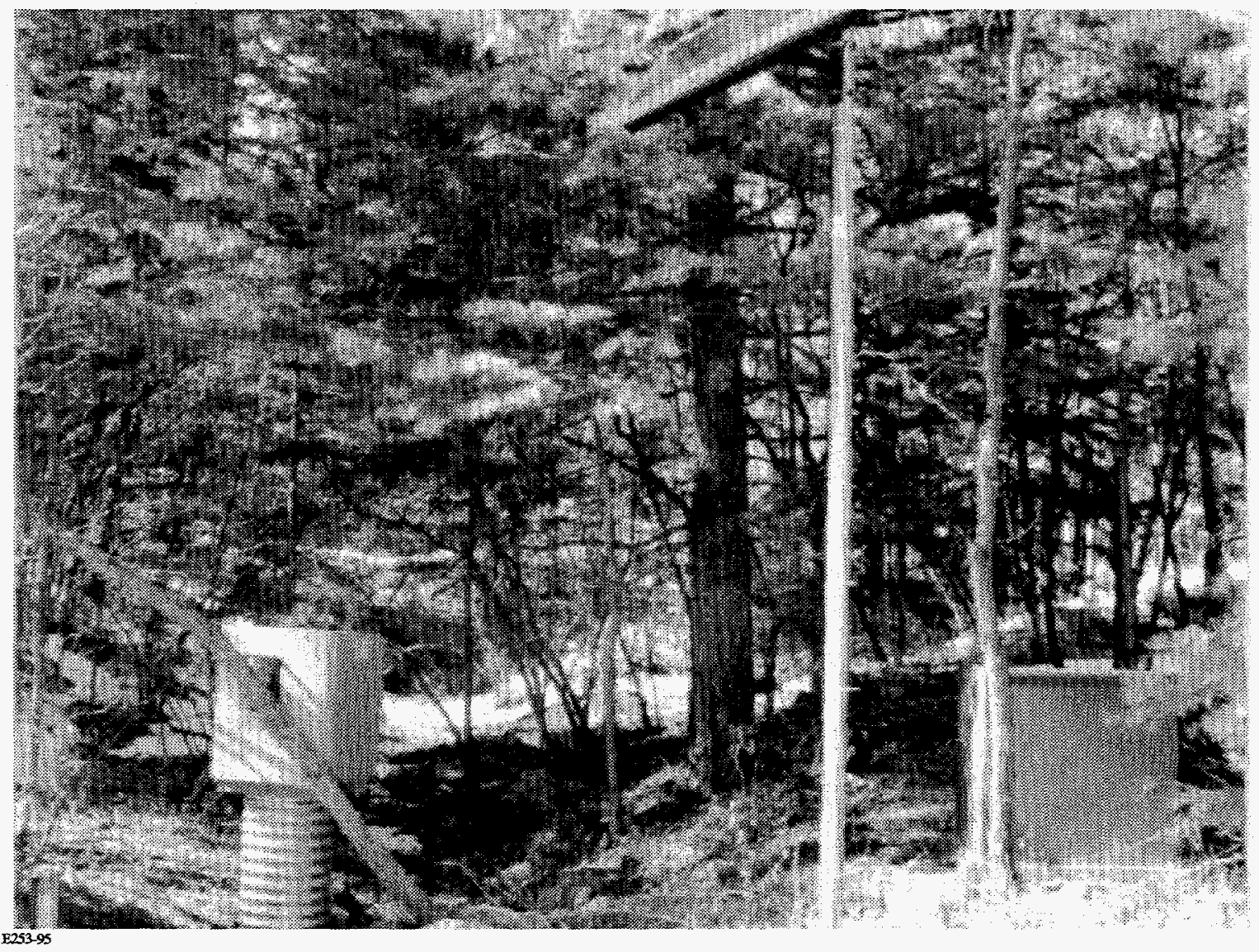




\section{Cañon del Valle above Highway 501 near Los Alamos, NM}

DAILY DISCHARGE IN CUBIC FEET PER SECOND

WATER YEAR OCTOBER 1994 TO SEPTEMBER 1995

\begin{tabular}{|c|c|c|c|c|c|c|c|c|c|c|c|c|}
\hline Day & OCT & NOV & DEC & JAN & FEB & MAR & APR & MAY & $\pi N$ & $\mathrm{JUL}$ & AUG & SEP \\
\hline 1 & 0 & 0 & 0 & 0 & 0 & 0 & 0 & 0 & 0 & 0 & 0 & 0 \\
\hline 2 & 0 & 0 & 0 & 0 & 0 & 0 & 0 & 0 & 0 & 0 & 0 & 0 \\
\hline 3 & 0 & 0 & 0 & 0 & 0 & 0 & 0 & 0 & 0 & 0 & 0 & 0 \\
\hline 4 & 0 & 0 & 0 & 0 & 0 & 0 & 0 & 0 & 0 & 0 & 0 & 0 \\
\hline 5 & 0 & 0 & 0 & 0 & 0 & 0 & 0 & 0 & 0 & 0 & 0 & 0 \\
\hline 6 & 0 & 0 & 0 & 0 & 0 & 0 & 0 & 0 & 0 & 0 & 0 & 0 \\
\hline 7 & 0 . & 0 & 0 & 0 & 0 & 0 & 0 & 0 & 0 & 0 & 0 & 0 \\
\hline 8 & 0 & 0 & 0 & 0 & 0 & 0 & 0 & 0 & 0 & 0 & 0 & 0 \\
\hline 9 & 0 & 0 & 0 & 0 & 0 & 0 & 0 & 0 & 0 & 0 & 0 & 0 \\
\hline 10 & 0 & 0 & 0 & 0 & 0 & 0 & 0 & 0 & 0 & 0 & 0 & 0 \\
\hline 11 & 0 & 0 & 0 & 0 & 0 & 0 & 0 & 0 & 0 & 0 & 0 & 0 \\
\hline 12 & 0 & 0 & 0 & 0 & 0 & 0 & 0 & 0 & 0 & 0 & 0 & 0 \\
\hline 13 & 0 & 0 & 0 & 0 & 0 & 0 & 0 & 0 & 0 & 0 & 0 & 0 \\
\hline 14 & 0 & 0 & 0 & 0 & 0 & 0 & 0 & 0 & 0 & 0 & 0 & 0 \\
\hline 15 & 0 & 0 & 0 & 0 & 0 & 0 & 0 & 0 & 0 & 0 & 0 & 0 \\
\hline 16 & 0 & 0 & 0 & 0 & 0 & 0 & 0 & 0 & 0 & 0 & 0 & 0 \\
\hline 17 & 0 & 0 & 0 & 0 & 0 & 0 & 0 & 0 & 0 & 0 & 0 & 0 \\
\hline 18 & 0 & 0 & 0 & 0 & 0 & 0 & 0 & 0 & 0 & 0 & 0 & 0 \\
\hline 19 & 0 & 0 & 0 & 0 & 0 & 0 & 0 & 0 & 0 & 0 & 0 & 0 \\
\hline 20 & 0 & 0 & 0 & 0 & 0 & 0 & 0 & 0 & 0 & 0 & 0 & 0 \\
\hline 21 & 0 & 0 & 0 & 0 & 0 & 0 & 0 & 0 & 0 & 0 & 0 & 0 \\
\hline 22 & 0 & 0 & 0 & 0 & 0 & 0 & 0 & 0 & 0 & 0 & 0 & 0 \\
\hline 23 & 0 & 0 & 0 & 0 & 0 & 0 & 0 & 0 & 0 & 0 & 0 & 0 \\
\hline 24 & 0 & 0 & 0 & 0 & 0 & 0 & 0 & 0 & 0 & 0 & 0 & 0 \\
\hline 25 & 0 & 0 & 0 & 0 & 0 & 0 & 0 & 0 & 0 & 0 & 0 & 0 \\
\hline 26 & 0 & 0 & 0 & 0 & 0 & 0 & 0 & 0 & 0 & 0 & 0 & 0 \\
\hline 27 & 0 & 0 & 0 & 0 & 0 & 0 & 0 & 0 & 0 & 0 & 0 & 0 \\
\hline 28 & 0 & 0 & 0 & 0 & 0 & 0 & 0 & 0 & 0 & 0 & 0 & 0 \\
\hline 29 & 0 & 0 & 0 & 0 & $\ldots$ & 0 & 0 & 0 & 0 & 0 & 0 & 0 \\
\hline 30 & 0 & 0 & 0 & 0 & - & 0 & 0 & 0 & 0 & 0 & 0 & 0 \\
\hline 31 & 0 & - & 0 & 0 & - & 0 & $=$ & 0 & $\longrightarrow$ & 0 & 0 & $\ldots$ \\
\hline Total & 0 & 0 & 0 & 0 & 0 & 0 & 0 & 0 & 0 & 0 & 0 & 0 \\
\hline Mean & 0 & 0 & 0 & 0 & 0 & 0 & 0 & 0 & 0 & 0 & 0 & 0 \\
\hline $\operatorname{Max}$ & 0 & 0 & 0 & 0 & 0 & 0 & 0 & 0 & 0 & 0 & 0 & 0 \\
\hline Min & 0 & 0 & 0 & 0 & 0 & 0 & 0 & 0 & 0 & 0 & 0 & 0 \\
\hline AC-FT & 0 & 0 & 0 & 0 & 0 & 0 & 0 & 0 & 0 & 0 & 0 & 0 \\
\hline
\end{tabular}

CY 1994: TOTAL* 0 MEAN 0 MAX 0 MIN 0 AC-FT 0

WATER YEAR 1995: TOTAL 0 MEAN 0 MAX 0 MIN

0 AC-FT 0

* Incomplete Record 


\section{Potrillo Canyon near White Rock, NM}

LOCATION. Lat $35^{\circ} 48^{\prime} 53^{\prime \prime}$, long $106^{\circ} 14^{\prime} 00^{\prime \prime}$, in Ramon Vigil Grant, Los Alamos County, 2.0 mi SW of White Rock and 0.25 mi upstream from NM State Highway 4.

DRAINAGE AREA. $2.25 \mathrm{mi}^{2}$.

PERIOD OF RECORD. October 1993 to September 30, 1995.

GAGE. Data logger with cellular telemetry and concrete control. Elevation of gage is $6,460 \mathrm{ft}$ above National Geodetic Vertical Datum of 1929 from topographic map.

REMARKS. Records good to fair.

EXTREMES FOR PERIOD OF RECORD. Maximum discharge $63 \mathrm{cfs}$ Aug. 29, 1995, gage height $2.70 \mathrm{ft}$. No flow most of time.

EXTREMES FOR CURRENT WATER YEAR. Maximum discharge $63 \mathrm{cfs}$ at $1355 \mathrm{hrs,} \mathrm{Aug.}$ 29, 1995, gage height $2.70 \mathrm{ft}$. No flow most of time.

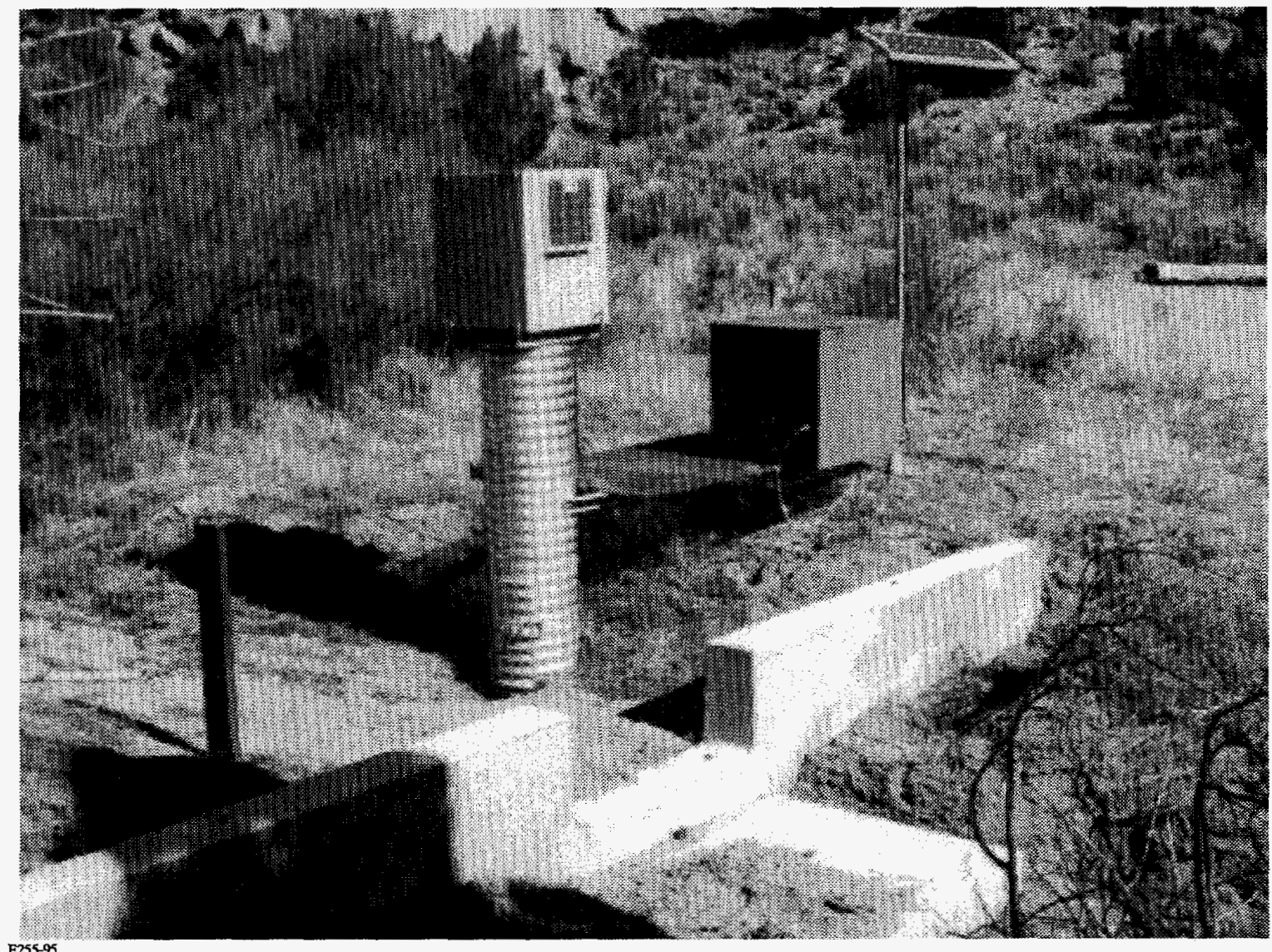


8313255 Potrillo Canyon near White Rock, NM

DAILY DISCHARGE IN CUBIC FEET PER SECOND

WATER YEAR OCTOBER 1994 TO SEPTEMBER 1995

\begin{tabular}{|c|c|c|c|c|c|c|c|c|c|c|c|c|}
\hline Day & OCT & NOV & DEC & JAN & FEB & MAR & APR & MAY & JUN & JUL & AUG & SEP \\
\hline 1 & 0 & 0 & 0 & 0 & 0 & 0 & 0 & 0 & 0 & 0 & 0 & 0 \\
\hline 2 & 0 & 0 & 0 & 0 & 0 & 0 & 0 & 0 & 0 & 0 & 0 & 0 \\
\hline 3 & 0 & 0 & 0 & 0 & 0 & 0 & 0 & 0 & 0 & 0 & 0 & 0 \\
\hline 4 & 0 & 0 & 0 & 0 & 0 & 0 & 0 & 0 & 0 & 0 & 0 & 0 \\
\hline 5 & 0 & 0 & 0 & 0 & 0 & 0 & 0 & 0 & 0 & 0 & 0 & 0 \\
\hline 6 & 0 & 0 & 0 & 0 & 0 & 0 & 0 & 0 & 0 & 0 & 0 & 0 \\
\hline 7 & 0 & 0 & 0 & 0 & 0 & 0 & 0 & 0 & 0 & 0 & 0 & 0.34 \\
\hline 8 & 0 & 0 & 0 & 0 & 0 & 0 & 0 & 0 & 0 & 0 & 0 & 0.03 \\
\hline 9 & 0 & 0 & 0 & 0 & 0 & 0 & 0 & 0 & 0 & 0 & 0 & 0 \\
\hline 10 & 0 & 0 & 0 & 0 & 0 & 0 & 0 & 0 & 0 & 0 & 0 & 0 \\
\hline 11 & 0 & 0 & 0 & 0 & 0 & 0 & 0 & 0 & 0 & 0 & 0 & 0 \\
\hline 12 & 0 & 0 & 0 & 0 & 0 & 0 & 0 & 0 & 0 & 0 & 0 & 0 \\
\hline 13 & 0 & 0 & 0 & 0 & 0 & 0 & 0 & 0 & 0 & 0 & 0 & 0 \\
\hline 14 & 0 & 0 & 0 & 0 & 0 & 0 & 0 & 0 & 0 & 0 & 0 & 0 \\
\hline 15 & 0 & 0 & 0 & 0 & 0 & 0 & 0 & 0 & 0 & 0 & 0 & 0 \\
\hline 16 & 0 & 0 & 0 & 0 & 0 & 0 & 0 & 0 & 0 & 0 & 0 & 0 \\
\hline 17 & 0 & 0 & 0 & 0 & 0 & 0 & 0 & 0 & 0 & 0 & 0 & 0 \\
\hline 18 & 0 & 0 & 0 & 0 & 0 & 0 & 0 & 0 & 0 & 0 & 0 & 0 \\
\hline 19 & 0 & 0 & 0 & 0 & 0 & 0 & 0 & 0 & 0 & 0 & 0 & 0 \\
\hline 20 & 0 & 0 & 0 & 0 & 0 & 0 & 0 & 0 & 0 & 0 & 0 & 0 \\
\hline 21 & 0 & 0 & 0 & 0 & 0 & 0 & 0 & 0 & 0 & 0 & 0 & 0 \\
\hline 22 & 0 & 0 & 0 & 0 & 0 & 0 & 0 & 0 & 0 & 0 & 0 & 0 \\
\hline 23 & 0 & 0 & 0 & 0 & 0 & 0 & 0 & 0 & 0 & 0 & 0 & 0 \\
\hline 24 & 0 & 0 & 0 & 0 & 0 & 0 & 0 & 0 & 0 & 0 & 0 & 0 \\
\hline 25 & 0 & 0 & 0 & 0 & 0 & 0 & 0 & 0 & 0 & 0 & 0 & 0 \\
\hline 26 & 0 & 0 & 0 & 0 & 0 & 0 & 0 & 0 & 0 & 0 & 0 & 0 \\
\hline 27 & 0 & 0 & 0 & 0 & 0 & 0 & 0 & 0 & 0 & 0 & 0 & 0 \\
\hline 28 & 0 & 0 & 0 & 0 & 0 & 0 & 0 & 0 & 0 & 0 & 0 & 0 \\
\hline 29 & 0 & 0 & 0 & 0 & - & 0 & 0 & 0 & 0 & 0 & 1.4 & 0 \\
\hline 30 & 0 & 0 & 0 & 0 & - & 0 & 0 & 0 & 0 & 0 & 0 & 0 \\
\hline 31 & 0 & $\ldots$ & 0 & 0 & 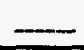 & 0 & - & 0 & - & 0 & 0 & - \\
\hline Total & 0 & 0 & 0 & 0 & 0 & 0 & 0 & 0 & 0 & 0 & 1.4 & 0.37 \\
\hline Mean & 0 & 0 & 0 & 0 & 0 & 0 & 0 & 0 & 0 & 0 & 0.045 & 0.012 \\
\hline $\operatorname{Max}$ & 0 & 0 & 0 & 0 & 0 & 0 & 0 & 0 & 0 & 0 & 1.4 & 0.34 \\
\hline Min & 0 & 0 & 0 & 0 & 0 & 0 & 0 & 0 & 0 & 0 & 0 & 0 \\
\hline AC-FT & 0 & 0 & 0 & 0 & 0 & 0 & 0 & 0 & 0 & 0 & 2.8 & 0.7 \\
\hline
\end{tabular}

CY 1994: TOTAL* 0 MEAN 0 MAX 0 MIN 0 AC-FT 0

WATER YEAR 1995: TOTAL 1.77 MEAN 0.005 MAX 1.4 MIN 0 AC-FT 3.5

* Incomplete Record 


\section{Los Alamos Canyon Seepage/Channel Loss Investigation}

Reach. Investigation was done from just below Los Alamos Reservoir to the mouth of the canyon. Locations are referenced to two physical features 1) gaging station 08313025 and 2) the security fence at the downstream boundary of Los Alamos Technical Area (TA) 2.

Date. All discharge measurements were made May 3, 1995.

Weather. Weather for the data collection effort was excellent. There was no precipation before or during the investigation to bias the results. Temperatures were at or near normal.

Streamflow. Flow in the reach was steady from spillage from Los Alamos Reservoir. The following table summarizes the gains and losses. Increases from the only flowing tributary (Pueblo Canyon) is considered a contribution and not a gain from seepage.

Remarks. Seepage investigation is rated good based on weather, lack of precipitation, and favorable discharge measuring conditions. Individual discharge measurements are rated good $\pm 5 \%$ and accuracy should be considered when evaluating results of this investigation.

Date: May 3, 1995

\begin{tabular}{|c|c|c|c|c|c|c|c|}
\hline \multirow[b]{2}{*}{ Stream } & \multirow[b]{2}{*}{ Location } & \multirow[b]{2}{*}{ Time } & \multirow{2}{*}{$\begin{array}{l}\text { Water } \\
\text { Temp } \\
\left({ }^{\circ} \mathrm{C}\right)\end{array}$} & \multirow{2}{*}{$\begin{array}{l}\text { Specific } \\
\text { Conductance } \\
\text { Us/cm }\end{array}$} & \multicolumn{3}{|c|}{ Discharge } \\
\hline & & & & & $\mathrm{ft}^{3 / \mathrm{s}}$ & $\begin{array}{l}\text { Gain/Loss } \\
\text { percent }\end{array}$ & $\begin{array}{l}\text { Gain/Loss } \\
\mathrm{ft}^{3} / \mathrm{s}\end{array}$ \\
\hline $\begin{array}{l}\text { Los Alamos } \\
\text { Canyon }\end{array}$ & $\begin{array}{l}0.5 \mathrm{mi} \mathrm{U} / \mathrm{S} \text { from } \\
\text { Gage } 08313025 \\
\text { Lat } 35^{\circ} 52^{\prime} 45^{\prime \prime} \text {, long } 106^{\circ} 20^{\prime} 14^{\prime \prime}\end{array}$ & 1020 & 6.0 & 120 & 5.51 & - & - \\
\hline $\begin{array}{l}\text { Los Alamos } \\
\text { Canyon }\end{array}$ & $\begin{array}{l}\text { Below Rainbow Bridge from } \\
\text { Gage } 08313025 \\
\text { Lat } 35^{\circ} 52^{\prime} 48^{\prime \prime} \text {, long } 106^{\circ} 19^{\prime} 42^{\prime \prime}\end{array}$ & 1130 & 6.5 & 110 & 5.51 & 0 & $\mathbf{0}$ \\
\hline $\begin{array}{l}\text { Los Alamos } \\
\text { Canyon }\end{array}$ & Lat $35^{\circ} 52^{\prime} 50^{\prime \prime}$, long $106^{\circ} 19^{\prime} 12^{\prime \prime}$ & 1215 & 7.0 & 100 & 5.24 & -4.9 & -.27 \\
\hline $\begin{array}{l}\text { Los Alamos } \\
\text { Canyon }\end{array}$ & $\begin{array}{l}1.0 \mathrm{mi} \text { below Gage } 08313025 \\
\text { Lat } 35^{\circ} 52^{\prime} 45^{\prime \prime} \text {, long } 106^{\circ} 18^{\prime} 42^{\prime \prime}\end{array}$ & 1210 & 8.3 & 110 & 6.33 & +20.8 & +1.09 \\
\hline $\begin{array}{l}\text { Los Alamos } \\
\text { Canyon }\end{array}$ & $\begin{array}{l}1.5 \mathrm{mi} \mathrm{D/S} \text { from Gage } 08313025 \\
\text { Lat } 35^{\circ} 52^{\prime} 37^{\prime \prime} \text {, long } 106^{\circ} 18^{\prime} 17^{\prime \prime}\end{array}$ & 1120 & 7.8 & 110 & 6.54 & +3.3 & +.21 \\
\hline $\begin{array}{l}\text { Los Alamos } \\
\text { Canyon }\end{array}$ & $\begin{array}{l}1.8 \mathrm{mi} \mathrm{D} / \mathrm{S} \text { from Gage } 08313025 \\
\text { Lat } 35^{\circ} 52^{\prime} 34^{\prime \prime} \text {, long } 106^{\circ} 17^{\prime} 58^{\prime \prime}\end{array}$ & 1040 & 7.3 & 110 & 6.75 & +3.2 & +.21 \\
\hline $\begin{array}{l}\text { Los Alamos } \\
\text { Canyon }\end{array}$ & $\begin{array}{l}2.2 \mathrm{mi} \mathrm{D} / \mathrm{S} \text { from Gage } 08313025 \\
\text { Lat } 35^{\circ} 52^{\prime} 32^{\prime \prime} \text {, long } 106^{\circ} 17^{\prime} 39^{\prime \prime}\end{array}$ & 1000 & 6.5 & 120 & 6.79 & +0.6 & +.07 \\
\hline $\begin{array}{l}\text { Los Alamos } \\
\text { Canyon }\end{array}$ & $\begin{array}{l}\text { At security fence just D/S TA-2 } \\
\text { Lat } 35^{\circ} 52^{\prime} 34^{\prime \prime} \text {, long } 106^{\circ} 17^{\prime} 24^{\prime \prime}\end{array}$ & 1025 & 7.0 & 130 & 6.96 & +2.5 & +.17 \\
\hline
\end{tabular}


Date: May 3, 1995

\begin{tabular}{|c|c|c|c|c|c|c|c|}
\hline \multirow[b]{2}{*}{ Stream } & \multirow[b]{2}{*}{ Location } & \multirow[b]{2}{*}{ Time } & \multirow{2}{*}{$\begin{array}{l}\text { Water } \\
\text { Temp } \\
\left({ }^{\circ} \mathrm{C}\right)\end{array}$} & \multirow{2}{*}{$\begin{array}{l}\text { Specific } \\
\text { Conductance } \\
\text { Us/cm }\end{array}$} & \multicolumn{3}{|c|}{ Discharge } \\
\hline & & & & & $\mathrm{ft}^{3 / \mathrm{s}}$ & $\begin{array}{l}\text { Gain/Loss } \\
\text { percent }\end{array}$ & $\begin{array}{l}\text { Gain/Loss } \\
\mathrm{ft}^{3 / 5}\end{array}$ \\
\hline $\begin{array}{l}\text { Los Alamos } \\
\text { Canyon }\end{array}$ & $\begin{array}{l}0.5 \mathrm{mi} \mathrm{D} / \mathrm{S} \text { from TA-2 fence } \\
\text { Lat } 35^{\circ} 52^{\prime} 27^{\prime \prime} \text {, long } 106^{\circ} 17^{\prime} 24^{\prime \prime}\end{array}$ & 1110 & 8.5 & 120 & 6.44 & -7.5 & -.52 \\
\hline $\begin{array}{l}\text { Los Alamos } \\
\text { Canyon }\end{array}$ & $\begin{array}{l}1.0 \mathrm{mi} \text { D/S from TA-2 fence } \\
\text { Lat } 35^{\circ} 52^{\prime} 24^{\prime \prime} \text {, long } 106^{\circ} 16^{\prime} 20^{\prime \prime}\end{array}$ & 1215 & 9.5 & 120 & 6.77 & +5.1 & +.33 \\
\hline $\begin{array}{l}\text { Los Alamos } \\
\text { Canyon }\end{array}$ & $\begin{array}{l}1.7 \mathrm{mi} \mathrm{D} / \mathrm{S} \text { from TA-2 fence } \\
\text { at Gage } 08313030 \\
\text { Lat } 35^{\circ} 52^{\prime} 20^{\prime \prime}, \text { long } 106^{\circ} 5^{\prime} 37^{\prime \prime}\end{array}$ & 1245 & 11.0 & 130 & 6.18 & -8.8 & -.59 \\
\hline $\begin{array}{l}\text { Los Alamos } \\
\text { Canyon }\end{array}$ & $\begin{array}{l}2.2 \mathrm{mi} D / S \text { from TA-2 fence } \\
\text { Lat } 35^{\circ} 52^{\prime} 24^{\prime \prime}, \text { long } 106^{\circ} 15^{\prime} 8^{\prime \prime}\end{array}$ & 1335 & 11.5 & 130 & 5.85 & -5.3 & -.33 \\
\hline $\begin{array}{l}\text { Los Alamos } \\
\text { Canyon }\end{array}$ & $\begin{array}{l}2.8 \mathrm{mi} \mathrm{D} / \mathrm{S} \text { from TA-2 fence } \\
\text { Lat } 35^{\circ} 52^{\prime} 10^{\prime \prime}, \text { long } 106^{\circ} 14^{\prime} 28^{\prime \prime}\end{array}$ & 1415 & 13.0 & 140 & 5.74 & -1.9 & -.11 \\
\hline $\begin{array}{l}\text { Los Alamos } \\
\text { Canyon }\end{array}$ & $\begin{array}{l}3.4 \mathrm{mi} \mathrm{D} / \mathrm{S} \text { from TA-2 fence } \\
\text { Lat } 35^{\circ} 52^{\prime} 8^{\prime \prime}, \text { long } 106^{\circ} 13 ' 55^{\prime \prime}\end{array}$ & 1405 & 12.6 & 140 & 5.26 & -8.4 & -.48 \\
\hline $\begin{array}{l}\text { Los Alamos } \\
\text { Canyon }\end{array}$ & $\begin{array}{l}\text { Los Alamos Canyon } \\
\text { near Los Alamos } \\
\text { Gage } 08313042 \\
\text { Lat } 35^{\circ} 52^{\prime} 01^{\prime \prime} \text {, long } 106^{\circ} 13^{\prime} 21^{\prime \prime}\end{array}$ & 1330 & 13.4 & 140 & 4.76 & -9.5 & -.50 \\
\hline Pueblo Canyon & $\begin{array}{l}\text { Pueblo Canyon } \\
\text { Gage } 08313060 \\
\text { Lat } 35^{\circ} 52^{\prime} 13^{\prime \prime} \text {, long } 106^{\circ} 12^{\prime} 56^{\prime \prime}\end{array}$ & 1530 & 22.0 & - & 0.87 & "In Flow" & \\
\hline $\begin{array}{l}\text { Los Alamos } \\
\text { Canyon }\end{array}$ & $\begin{array}{l}\text { At mouth } 100 \mathrm{ft} \mathrm{D} / \mathrm{S} \\
\text { from Rio Grande at Otowi } \\
\text { Gage } 08313030 \\
\text { Lat } 35^{\circ} 52^{\prime} 29^{\prime \prime}, \text { long } 106^{\circ} 8^{\prime} 30^{\prime \prime}\end{array}$ & 24.0 & 230 & 1.35 & -76.0 & -4.28 & \\
\hline
\end{tabular}

